\title{
L'Espagne et le droit de la mer
}

\author{
VALENTÍN BOU ET ROMUALDO BERMEJO
}

\section{INTRODUCTION}

Pour l'Etat espagnol, le droit de la mer et les nouvelles tendances qui apparaissent dans ce domaine dans la pratique des Etats ont une importance spéciale. Cette importance est due à des considérations géographiques, stratégiques et économiques. Parmi les considérations géographiques, il convient de rappeler que l'Etat espagnol possède un littoral maritime d'environ $7.880 \mathrm{~km}$ de côtes comprenant le territoire péninsulaire, les deux archipels et les villes de Ceuta et Melilla. Par ailleurs, l'Espagne est un Etat riverain d'une mer semi-fermée, la Méditérranée, et d'un océan, l'Atlantique, dans lesquels le droit de la mer présente d'importants développements normatifs au niveau régional.

Les motifs stratégiques qui confirment l'importance essentielle pour l'Espagne du droit de la mer, comprennent entre autres, le fait qu'il s'agit d'un Etat riverain de deux détroits utilisés pour la navigation internationale, le canal de Minorque et le détroit de Gibraltar. Ce dernier, étant une des deux voies qui font communiquer la Mer Méditérranée avec des mers plus vastes, est une des voies de navigation dont le trafic maritime est le plus important du monde. On peut mentionner aussi le fait que l'Espagne exerce la souveraineté sur deux importants ensembles d'archipels, l'archipel des Baléares et celui des Canaries (ce dernier étant un point important du système intercontinental de communication), ou le fait également remarquable que les diverses zones sous souveraineté espagnole dans le nord de l'Afrique sont toutes riveraines de la Mer Méditérranée.

Enfin, au nombre des considérations économiques, il faut rappeler que: l'Espagne est l'une des premières puissances mondiales en matière de pêche maritime, en particulier grâce à sa flotte de haute mer; que l'importante industrie touristique espagnole repose principalement sur son vaste littoral maritime, ce qui explique la sensibilité spéciale de l'Espagne pour la préservation et la protection de l'environnement marin; que l'Espagne est déficitaire en plusieurs ressources, présentes cependant dans la zone des hauts fonds marins et océaniques dont l'exploitation commerciale devrait commencer dans un avenir plus ou moins proche, etc.

Ces facteurs, avec d'autres, ont porté l'Etat espagnol à prendre une part active à chacune des conférences de codification du droit de la mer, organisées par la Société des Nations et par l'Organisation des Nations Unies, 
ainsi qu'aux différentes tentatives codificatrices régionales concernant les mers dont l'Espagne est riveraine.

Il n'en reste pas moins que, jusqu'au début de la décade des années 70 , la réglementation espagnole en matière de droit de la mer était insuffisante, hétérogène et conjoncturelle. Depuis la Cellule Royale du Conseil des Finances du 17 décembre 1760 fixant la largeur des eaux territoriales à 6 m.m. pour la répression de la contrebande du tabac et du sel, ${ }^{1}$ les zones maritimes espagnoles restèrent pratiquement intactes jusqu'à il y a peu de temps. Parallèlement à l'adhésion de l'Espagne aux quatre conventions adoptées à Genève le 29 avril 1958, résultant de la I CNUDM, ${ }^{2}$ une importante activité normative espagnole s'est développée dans ce domaine, rejoignant en grande partie, bien que non entièrement, le résultat des travaux de la III CNUDM.

De manière générale, on peut affirmer que même si les quatre conventions résultant de la I CNUDM favorisaient et protégeaient convenablement les intérêts maritimes de l'Espagne, diverses prétentions juridiques qui apparurent au cours de la III CNUDM, et qui plus tard furent reprises dans la Convention de Montego Bay, en particulier celles relatives au régime de la navigation internationale par les détroits, ont été considérées par l'Espagne comme extrêmement préjudiciables à ses intérêts nationaux. De ce fait, la délégation espagnole s'est abstenue dans le vote de la Convention de Montego Bay et, au moment d'expliquer cette abstention, M. Lacleta Muñoz, représentant de la délégation espagnole, formula la déclaration suivante:

“... consciente de l'importance politique et historique de la clôture des travaux de la Conférence, la délégation espagnole s'est bornée à s'abstenir, bien que sa position sur une question très importante la concernant très directement, n'ait pas été reflétée de manière adéquate dans le texte adopté. Il s'agit de la troisième partie du projet de convention et, plus particulièrement, des articles $38,39,41$ et 42 . La position de l'Espagne sur la question est consignée dans le document A/CONF.62/L.136. En outre, le Gouvernement espagnol fait objection de manière générale au texte de la résolution III et notamment au paragraphe 2 de celle-ci.

Enfin, en ce qui concerne tout au moins les questions susmentionnées, les textes adoptés par la Conférence ne constituent pas, à ses yeux, une codification du droit coutumier...". ${ }^{3}$

1 Reproduite dans: DE YTURRIAGA BARBERAN, Textos $y$ documentos, in POCH (sous la direction de), La actual revisión del Derecho del Mar. Una perspectiva española, 1975, II, 1, p. 47.

2 L'Etat espagnol se rallia aux quatre conventions de Genève du 29 avril 1958. Le 25 décembre 1971, la Convention de 1958 sur la mer territoriale fut publié officiellement dans le B.O.E.. La Convention de 1958 sur le plateau continental dans le B.O.E. du 25 décembre 1971. La Convention de 1958 sur la haute mer dans le B.O.E. du 27 décembre 1971 et la Convention de 1958 sur la pêche et la conservation des ressources de la haute mer dans le B.O.E. du 27 décembre 1971.

3 Cfr. III CNUDM, Documentos oficiales, XVI, p. 166, par. 99-100. 
Ce qui précède, ajouté à d'autres facteurs, et à une signature de la Convention de Montego Bay vraiment tardive (le 5 décembre 1984, quelques jours avant l'échéance du délai prévu) et accompagnée de déclarations interprétatives rappelant la position de l'Espagne à l'egard du régime de passage en transit par les détroits internationaux, ${ }^{4}$ expliquent pourquoi l'Espagne n'a pas ratifié la Convention de Montego Bay au cours des années suivant 1984.

A la différence d'autres Etats industrialisés, l'Espagne n'a pas rejeté ouvertement la Partie XI de la Convention de Montego Bay. L'adoption le 29 juin 1994 d'un Accord relatif à l'application de la Partie XI de la Convention des Nations Unies sur le droit de la mer qui amende cette Partie XI, n'en peut que modifier complètement l'attitude espagnole envers la Convention de Montego Bay. Cela n'est pas dû au fait que cet amendement à la Partie XI de la Convention de Montego Bay peut favoriser réellement les intérêts espagnols dans cet espace maritime. Cela dépend de l'impossibilité pratique pour l'Espagne de maintenir sa thèse sur la navigation à travers les détroits internationaux dans l'hypothèse d'une ratification massive de la Convention de Montego Bay, en particulier de la part des autres Etats industrialisés. C'est peut-être pour cette raison que dans un futur proche le problème de la ratification de la Convention de Montego Bay par l'Espagne se posera à nouveau.

\section{LIGNES DE BASE}

\subsection{Le système espagnol des lignes de base}

Appliquant une législation qui remonte à 1962,5 le décret royal 2510/1977 du 5 août 6 établit le système des lignes de bases droites pour la délimitation des zones maritimes espagnoles. Sur la quasi totalité du littoral continental espagnol, avec les importantes exceptions de Gibraltar ${ }^{7}$ et des territoires de

\footnotetext{
4 Ces déclarations ont été publiées dans 4 Bull. D.M., 1985, p. 15.

5 L'art. 1 de la loi 93/1962 du 24 décembre à propos de sanctions pour les infractions commises par les navires étrangers en matière de pêche (B.O.E. du 25 décembre 1962) prévoyait la possibilité de tracer des lignes de bases droites d'environ 24 m.m. pour les baies; l'art. 2 de la loi 20/1967 du 8 avril sur l'extension des eaux territoriales espagnoles à $12 \mathrm{~m} . \mathrm{m}$. dans un but de pêche (B.O.E. du 11 avril 1967) généralisa cette mesure à toutes les côtes espagnoles.

6 B.O.E. du 30 septembre 1977. Correction des erreurs du B.O.E. du 20 octobre 1977. Ce décret abrogea un précédent décret portant sur le même thème daté du 5 mars 1976 (B.O.E. du 30 mars 1976).

7 En adhérant à ces quatre Conventions de Genève de 1958, l'Espagne fit la déclaration suivante à toutes et à chacune d'elles: “.... cependant, son adhésion ne peut être interprétée comme une reconnaissance de n'importe quels droits ou situations relatives aux espaces maritimes de Gibraltar qui ne sont pas inclus dans l'article 10 du Traité d'Utrecht du 13 juillet 1713, entre les Couronnes d'Espagne et de Grande-Bretagne". Cette déclaration a été réaffirnnée par la disposition finale première de la loi 10/1977 du 4 janvier au sujet de la mer territoriale (B.O.E. du 8 janvier 1977), de même que par la déclaration interprétative formulée par l'Espagne au moment de la signature de la Convention de Montego Bay le 5 décembre 1984.
} 
souveraineté espagnole dans le nord de l'Afrique, des lignes de base droites ont été tracées. Ce système de lignes de base est complémentaire à l'Accord Hispano-Français du 14 juillet $1959^{8}$ sur la pêche dans le Bidasoa et dans la Baie de Higuer, ${ }^{9}$ par lequel les deux pays ont convenu de tracer une ligne de clôture de la baie.

Il faut aussi mettre l'accent sur le fait que ce décret établit pour les Iles Canaries et Baléares un système de lignes de bases droites, et non archipélagiques. Cependant, en accord avec la position de la délégation espagnole à la III CNUDM, favorable à l'application du principe archipélagique non seulement aux Etats archipélagiques, mais aussi aux archipels d'Etat, ${ }^{10}$ la loi 15/1978 du 20 février spécifie que:

"Dans le cas des archipels, la limite extérieure de la zone économique sera calculée à partir des lignes de base droites qui joignent les points extrêmes des îles et des îlots qui, respectivement, les composent, de façon à ce que le périmètre obtenu suive la configuration générale de chaque archipel" (art. 1.1).11

Le Gouvernement n'a pas, cependant, pris les mesures nécéssaires pour l'application de cette règle. 12

\subsection{Navigation dans les eaux intérieures}

Dans le système juridique espagnol, la navigation dans les eaux intérieures est réglementée par une législation très dispersée. Mis à part ce qui est prévu pour l'accès aux ports espagnols, la navigation dans ces eaux tend à s'uniformiser avec le régime de navigation établi pour la mer territoriale. Ainsi est-il que, à l'exception de l'accès aux ports, on n'a pas établi de distinction de zone entre le régime de la navigation dans les eaux intérieures et la mer territoriale espagnole.

\footnotetext{
8 Ceuta, Melilla, Iles Chafarinas, Ile du Peregil et les rochers de Vélez de la Gomera et de Alhucemas.

9 B.O.E. du 2 novembre 1965. Cet Accord abrogea la déclaration Hispano-Française relative à la délimitation des juridictions de Bidasoa et de la baie de Higuer du 30 mars 1879, bien qu'elle ait conservé la délimitation établie à cette même date. Malgré l'existence d'un régime conventionnel de longue date, on ne peut considérer qu'il s'agisse d'une baie historique commune aux deux Etats, car jamais elle ne fut réclamée en ce sens ni par l'Espagne ni par la France.

10 III CNUDM, Documentos oficiales, I, p. 193; II, p. 300; XVII, p. 92.

11 Loi 15/1978 du 20 février concernant la zone économique exclusive, B.O.E. du 23 novembre 1978.

12 Malgrè les prétentions des représentants politiques des Canaries au Sénat. Vide par ex., la proposition de loi présentée par le Groupe Parlementaire de la Coalition Canarienne de modification de la loi 15/1978 du 20 février au sujet de la zone économique qui établit dans son annexe 1 des lignes de base archipélagiques droites pour l'archipel des Canaries. Cfr. B.O.C.G., Senado, V Legislatura, Serie III, A: proposition de loi du Sénat 29 octobre 1993, n. 6 (a).
} 
En principe, l'entrée dans les ports espagnols ${ }^{13}$ des navires de commerce n'est pas soumise à autorisation, même si, pour des raisons de réglementation du trafic maritime ou de sécurité de la navigation, elle peut être soumise à des conditions par le Commandement de la Marine présente dans chaque port. ${ }^{14}$

Exceptionnellement, l'Administration pourra interdire, ou réduire, la navigation d'une certaine catégorie de navires de commerce dans les eaux intérieures, et même l'entrée au port, aussi bien pour éviter la réalisation d'activités illicites ou l'exercice de quelque trafic interdit, ${ }_{15}^{15}$ que dans le but de préserver la sécurité de la navigation et prévenir la pollution du milieu marin. ${ }^{16}$ Une réglementation spécifique régit l'entrée dans les ports espagnols des navires suivants: ceux qui transportent des substances dangereuses ou polluantes; ${ }^{17}$ des super-pétroliers; ${ }^{18}$ des navires de guerre étrangers, incluant tout navire transportant un Chef d'Etat ainsi que son cortège, à l'exception d'autres passagers en visite officielle; ${ }^{19}$ et des navires nucléaires,

13 Nous faisons uniquement référence aux ports d'intérêt général et aux ports commerciaux (art. 2, 3 et 5 de la loi 27/1992, du 24 novembre, sur les ports de l'Etat et de la Marine Marchande. B.O.E. du 25 novembre 1992. Nous ne tenons pas compte de l'étude des ports de refuge, des ports de plaisance et en général des ports dans lesquels il ne se déroule aucune activité commerciale, du fait qu'ils restent soumis à l'action normative de la respective Communauté Autonome d'après les art. 148.1.6 et 149.1.20 de la Constitution espagnole.

14 Voir par exemple, les fonctions du Commandement de la Marine dans l'art. 88.3 de la loi 27/1992 déjà citée.

is Ibidem, art. 111.

16 Ibidem, art. 112.

17 Voir la directive de la Communauté européenne relative aux conditions minimales exigées aux navires qui entrent ou sortent des ports de la Communauté et qui transportent des substances dangereuses ou polluantes (JOCE L 247 du 5 octobre 1993). Cette norme européenne n'a pas encore été transposée au droit espagnol, bien que cela paraît imminent.

18 Arrêté du 8 novembre 1990 par lequel on établit les conditions minimales de sécurité exigées pour les navires-citernes destinés au transport du pétrole et à ses dérivés, de gaz et de produits chimiques, de 1.600 tonneaux de jauge brut ou plus, qu'ils entrent ou qu'ils sortent des ports espagnols (B.O.E. du 19 novembre 1990). Cette norme fut adoptée dans le but d'augmenter la sécurité des eaux espagnoles et dans l'intention d'exécuter les directives 79/116 et 79/1.043 du Conseil des Communautés européennes sur les conditions minimales exigées pour les navires-citernes qui entrent ou qui sortent des ports maritimes de la Communauté, en accord avec la disposition additionnelle première du décret royal 145/1989 du 20 janvier par lequel on adopte le règlement d'admission, de manipulation, et de stockage de marchandises dangereuses dans les ports; B.O.E. du 13 novembre 1989.

19 Voir l'arrêté du Ministère de la Défense 25/1985 du 23 avril, par lequel on approuve les normes pour les escales de navires de guerre étrangers dans les ports ou mouillages espagnols, et leur passage par la mer territoriale espagnole en temps de paix (B.O.E. du 14 mai 1985). Ces normes ne s'appliquent pas, d'après l'art. 12, aux escales des navires d'armement ou de propulsion nucléaire, qui feront l'objet d'un accord spécial en chaque cas; aux navires de guerre bélligérants auxquels on appliquera les limitations en vigueur du droit international; aux navires de guerre appartenant aux nations avec lesquelles ont été établis, ou peuvent s'etablir, des accords ou conventions bilatérales en matière d'escales, qui seront régies par ces derniers, appliquant les normes de l'arrêté 25/1985 avec un caractère supplétoire. Ce dernier énoncé fut appliqué par la Convention entre le Royaume d'Espagne et les Etats-Unis d'Amérique sur la coopération pour la défense (Madrid, 1 décembre 1988), B.O.E. du 6 mai 1989. 
c'est-à-dire des navires avec armement, ou à propulsion nucléaire, incluant les navires de guerre qui réunissent ces conditions. ${ }^{20}$

\section{MER TERRITORIALE}

\subsection{Limites extérieures}

Malgré l'insistance de la diplomatie espagnole à affirmer que l'Espagne possède une mer territoriale de $6 \mathrm{~m} . \mathrm{m}$. depuis les temps de la Cellule Royale de 1760, dans le droit espagnol il n'existait aucune règle définissant à un niveau général le statut de cet espace maritime ou son extension. En ce qui concerne des activités très concrètes (douanes et répression de la contrebande, course maritime, neutralité, pêche) on avait fixé des étendues différentes des eaux territoriales espagnoles. Cette situation fut rectifiée par la loi 10/1977 du 4 janvier, concernant la mer territoriale. Cette loi déclare que la souveraineté de l'Etat espagnol s'étend à sa mer territoriale et s'exerce:

“... en conformité avec le droit international, sur la colonne d'eau, le lit, le fond marin et les ressources de cette mer, de même que l'espace aérien" (art. 1).

En outre l'art. 3, fixe la limite extérieure de la mer territoriale espagnole à 12 m.m., "étant donné que celle-ci est la limite établie actuellement par la majorité des Etats, et est considerée comme conforme au droit international en vigueur". ${ }^{21}$

\subsection{Passage inoffensif}

Les normes de la Convention de 1958 sur la mer territoriale qui réglementent le passage inoffensif en mer territoriale sont applicables à l'Espagne, puisqu'il s'agit d'un Etat partie contractante. Bien qu'aucune norme de droit interne ne donne application de façon générale à ces dispositions internationales, il existe une régle spécifique ayant trait aussi bien à des aspects

\footnotetext{
20 Voir les art. 73 à 77 de la loi 25/1964 du 29 avril régulatrice de l'energie nucléaire, B.O.E. du 4 mai 1964. Un régime beaucoup plus bénévole a été établi à un niveau bilatéral par la Convention entre le Royaume d'Espagne et les Etats-Unis d'Amérique au sujet de la coopération pour la défense citée plus haut, aussi bien pour les navires à propulsion nucléaire (ibidem, point 6 de l'annexe 3) que pour les navires à armement nucléaire (art. 11.2 de cette Convention). Voir à ce sujet l'arrêt du 5 juin 1993 de la Section Première de la salle du Contentieux Administratif de l'Audience Nationale.

21 Voir supra note 7. La tentative (même si par la suite elle a echoué dans la pratique) de cette loi d'en finir avec la pluralité des dénominations (eaux territoriales, zone maritime espagnole, eaux espagnoles, mer littorale nationale), que l'on utilisait dans le droit espagnol pour se référer à la mer territoriale, est louable.
} 
très concrets du passage inoffensif, qu'au passage de certains navires avec des caractéristiques spéciales.

C'est ainsi que, en premier lieu, le droit de passage inoffensif est reconnu seulement pour les navires et non pour les aéronefs. L'Accord sur l'aviation civile internationale (Chicago, 7 décembre 1944), 22 auquel la loi 48/1960 du 21 juillet sur la navigation aérienne donne exécution avec une terminologie très défectueuse, affirme que l'Etat espagnol pourra autoriser (ce qui est incompatible avec la jouissance du droit) le "passage inoffensif" sur son territoire des avions étrangers, à la suite de la conclusion d'un traité correspondant avec les autres Etats, ou par une autorisation spéciale. ${ }^{23}$ Cette disposition complète celle qui précise que "les aéronefs des Etats étrangers ne pourront pas voler au dessus du territoire sous la souveraineté nationale sans autorisation ou invitation préalable". ${ }^{24}$ La même solution a été établie pour les aéronefs nucléaires. 25

En second lieu, bien que les navires étrangers jouissent d'un droit de passage inoffensif dans la mer territoriale, l'Etat espagnol peut exercer sur ces navires d'importants pouvoirs de police. Le Gouvernement peut empêcher, réduire, ou soumettre à des conditions la navigation de certaines catégories de navires civils dans la mer territoriale espagnole afin d'empêcher la réalisation d'activités illicites ou l'exercice de quelque trafic interdit. ${ }^{26}$ En outre, afin de préserver la sécurité de la navigation et éviter la pollution du milieu marin dans la mer territoriale, les Autorités portuaires et les Commandements de la Marine peuvent visiter, inspecter, conditionner le mouillage, capturer, entamer des procédures judiciaires et, de façon générale, prendre les mesures considérées nécêssaires vis-à-vis des navires qui commettent ou sont susceptibles de commettre une infraction. ${ }^{27}$

22 B.O.E. du 24 février 1947 et du 29 décembre 1969.

23 Art. 2.2, B.O.E. du 23 décembre 1960.

24 Ibidem, art. 89. Une application pratique de cette normative se trouve dans l'art. 2.2 de la Convention du ler décembre 1988, entre le Royaume d'Espagne et les Etats-Unis d'Amérique, sur la coopération pour la défense, op. cit. Voir également ses art. 12 et 25 et ss. Une déscription des forces aériennes des Etats-Unis autorisées sur la base navale de Rota se trouve à l'Annexe 2, point 3 de cette Convention.

25 Art. 80 de la loi 25/1964 du 29 avril, règlementant l'énergie nucléaire citée supra, note 20. Cette solution coïncide avec celle qui a été établie de façon bilatérale dans les relations entre l'Espagne et les Etats-Unis, en tenant compte du fait qu'à cette occasion il fut précisé que: “... le fait que des aéronefs ayant un armement et du matériel nucléaire à bord ne survolent pas l'Espagne et que n'importe quel changement dans cette pratique exige le consentement du Gouvernement Espagnol, est la politique du Gouvernement Espagnol". Les Etats-Unis s'engagèrent à respecter entièrement cette politique. Voir l'Echange de notes du 1 er décembre 1988 entre les deux Etats sur la politique du Gouvernement Espagnol en matière de transits nucléaires, annexe à la Convention de la même date, entre le Royaume d'Espagne et les Etats-Unis D'Amérique, au sujet de la coopération pour la défense, op. cit.

26 Art. 111 de la loi 27/1992 du 24 novembre, des Ports de l'Etat et de la Marine Marchande, citée supra, note 13 .

27 Ibidem, art. 112. Il faut noter qu'à la différence de la disposition antérieure, l'art. 112 ne limite pas ces pouvoirs de police aux "navires civils". Cependant, vouloir exercer ces poụvoirs sur des navires d'Etat destinés à des fins non commerciales, ou sur des navires de guerre étrangers, serait, tout au moins, controversé dans la pratique, et en tout cas contraire aux art. 22 et 23 de la Convention de 1958 sur la mer territoriale et aux art. 30, 32 et 236 de la Convention de Montego Bay. 
En troisième lieu, et en anticipant sur la loi précitée, l'Espagne règlementa le mouillage des navires-citernes dans sa mer territoriale, ${ }^{28}$ donnant exécution de façon appropriée à l'art. 14.3 de la Convention de 1958 sur la mer territoriale. Dans le but de sauvegarder le trafic maritime et les milieux naturels, et de protéger l'environnement, le Ministère des Travaux Publics et des Transports a voulu mettre fin à la pratique suivie par de nombreux navires pétroliers de différentes nationalités, consistant à utiliser la zone du sud-ouest espagnol comme lieu de mouillage ${ }^{29}$ à durée illimitée, dans l'attente d'ordres définitifs de leur armateur concernant le point de déchargement où la cargaison sera le mieux côtée. On exige ainsi l'autorisation préalable de la Direction Générale de la Marine Marchande pour tous les navires-citerne, qui, n'ayant pas comme point de destination ou de déchargement de tout ou partie de leur cargaison un port ou un terminal espagnol, prétendent mouiller dans la mer territoriale espagnole, dans l'attente d'ordres ou d'instructions ou dans une circonstance quelconque qui se différencie du passage inoffensif défini à l'art. 14 de la Convention de 1958 sur la mer territoriale. La demande d'autorisation doit fournir une information très précise, portant notamment sur le type et la quantité de chargement et sur le temps prévu pour le séjour dans le mouillage. L'autorisation est soumise à la condition que le navire mouille effectivement dans la position géographique prescrite et qu'à son arrivée au mouillage il se soumette à une inspection de sécurité dont le résultat conditionnera l'autorisation de mouillage.

Enfin, l'Espagne a réglementé le passage dans sa mer territoriale de certains navires étrangers possédant des caractéristiques spéciales. Il s'agit, notamment, des navires de guerre et des navires nucléaires. En ce qui concerne le passage des navires de guerre étrangers dans sa mer territoriale, l'Etat espagnol a rapidement adhéré à la thèse défendue par les puissances occidentales, en particulier les Etats membres de l'OTAN, au cours de la négociation de la Convention de 1958 sur la mer territoriale. Selon ces Etats, il faut interpréter l'absence d'une disposition expresse réglementant le droit de passage inoffensif des navires de guerre dans cette Convention, dans le sens que ces navires sont inclus dans l'art. 14.1, à savoir que leur passage ne peut pas être interdit (en le soumettant à autorisation préalable) ni soumis à des conditions (en prévoyant la notification préalable). L'Espagne suivit précisément cette position au point 8 de l'arrêté du Ministère de la Marine 885/1958 du 29 mars, au sujet des visites des navires de guerre étrangers aux ports espagnols, et leur transit dans les eaux territoriales. ${ }^{30}$

\footnotetext{
28 Arrêté du 17 avril 1991 par lequel il est reglementé le mouillage des navires-citernes dans les eaux territoriales ou dans la ZEE; B.O.E. du 18 avril 1991.

29 Au moment d'approuver cet ordre, dans le Golfe de Cadiz six pétroliers mouillaient avec une cargaison de plus de 1.600 .000 tonnes de pétrole brut.

30 D'après cette disposition "en accord avec les normes coutumières du droit international, on ne requiert pas d'autorisation spéciale pour le transit dans les eaux territoriales espagnoles" des navires de guerre étrangers, B.O.E. du 31 mars 1958. Le 21 mars 1930, le délégué espagnol à la Conférence de La Haye avait défendu la thèse contraire. Voir DE YTURRIAGA BARBERAN, Textos... cit., supra note 1, II, 2, p. 394-397.
} 
Cette position, qui a prévalu à la III CNUDM, a été suivie à nouveau dans le droit espagnol postérieurement à l'adoption de la Convention de Montego Bay. Le point 11.1 de l'arrêté du Ministère de la Défense 25/1985 du 23 avril établit que:

“... il n'est pas requis d'autorisation spéciale pour le passage des navires de guerre étrangers dans la mer territoriale espagnole, dans laquelle ils sont tenus de respecter 'le passage inoffensif', en accord avec les normes coutumières du droit international...". ${ }^{31}$

Il faut ajouter que c'est uniquement à l'intention des navires de guerre que l'arrêté de 1985 réglemente une série d'aspects du droit de passage inoffensif, droit qui, en principe, vaut pour toutes les catégories de navires. Parmi ces points, se trouvent la définition du passage, 32 l'obligation pour les sous-marins de naviguer en surface et celle d'arborer leur pavillon de façon visible, 33 de même que la réglementation d'une série d'activités interdites aux navires de guerre étrangers lorsqu'ils naviguent dans la mer territoriale espagnole. Ces activités sont interdites par une réception incomplète dans le droit espagnol de l'art. 19.2 de la Convention de Montego Bay. Elles comprennent l'obligation de ne pas s'arrêter; d'amener les embarcations; de ne pas mettre en vol les aéronefs; de ne pas effectuer des manoeuvres, des exercices, des transbordements ou des transferts de n'importe quelle classe, ni même d'effectuer des travaux hydrographiques ou océanographiques. ${ }^{34}$ Cette dernière disposition complète celle qui établit que les navires de guerre étrangers, pour effectuer certains exercices ou tout autre opération en dehors du simple passage, doivent obtenir préalablement l'autorisation du Gouvernement espagnol, autorisation qui doit être sollicité par voie diplomatique. ${ }^{35}$

La réglementation de la navigation dans la mer territoriale espagnole des navires nucléaires étrangers, incluant les navires de guerre étrangers à armement ou à propulsion nucléaire, ${ }^{36}$ est beaucoup plus sévère. Conformément à l'art. 70 de la loi 25/1964 du 29 avril réglementant l'énergie nucléaire, le passage de ces navires dans la mer territoriale espagnole est considéré comme une exception au droit de passage inoffensif, le législateur espagnol étant de l'opinion que ces navires spéciaux ne sont pas titulaires de ce droit.

31 Voir supra, note 19. L'art. 2 de cet arrété annula l'ordre 885/1958 du 29 mars.

32 Ibidem, point 1.3 .

33 Ibidem, point 11.2. En réalité, cette disposition étend l'obligation d'arborer son pavillon à tous les navires de guerre, quand les art. 14.6 de la Convention de 1958 sur la mer territoriale et 20 de la Convention de Montego Bay l'exigent uniquement pour les sous-marins et autres véhicules submersibles, qu'ils soient de guerre ou non. Le point 13 de l'Annexe 3 de la Convention du ler décembre 1988 entre le Royaume d'Espagne et les Etats-Unis d'Amérique sur la coopération pour la défense est plus en accord avec ces dispositions intemationales.

34 Voir le point 11.2 de l'arrêté 25/1985, op. cit.

35 Ibidem, point 11.3 .

36 Ibidem, point 12 et art. 69 de la loi 25/1964 du 29 avril, réglementant l'énergie nucléaire, cit. supra note 20. 
On exige ainsi de la part des navires nucléaires le respect des normes internationales concernant le passage par la mer territoriale, ${ }^{37}$ en requérant également l'autorisation préalable du Gouvernement espagnol pour naviguer dans sa mer territoriale, où ils pourront être inspectés afin de vérifier leurs conditions de sécurité ainsi que leur fonctionnement. ${ }^{38}$ Cette norme paraît contraire au droit international de la mer.

L'Espagne n'a pas adopté une législation spécifique concernant le passage dans sa mer territoriale des navires étrangers qui transportent des substances nucléaires ou autres substances intrinsèquement dangereuses ou nocives. En réglementant les pouvoirs de police de l'Etat espagnol dans sa mer territoriale, les art. 111 et 112 de la loi 27/1992 du 24 novembre, sur les ports de l'Etat et la Marine Marchande 39 n'en ont pas moins délégué au Gouvernement la faculté d'adopter des règlements pour empêcher, réduire ou soumettre à des conditions la navigation de certains navires "civils" dans la mer territoriale espagnole, notamment afin de réprimer l'exercice de n'importe quel trafic interdit ou pour empêcher la pollution du milieu marin.

\section{DETROITS}

L'Espagne est un Etat riverain possédant deux détroits utilisés pour la navigation nationale: le détroit de Gibraltar et le canal de Minorque. $40 \mathrm{Il}$ est une constante traditionnelle dans le droit espagnol que sa législation sur la navigation dans la mer territoriale espagnole s'applique à toutes les parties de celle-ci, indépendamment du fait qu'il existe des parties de la mer territoriale espagnole qui constituent un détroit utilisé pour la navigation internationale. Ce principe d'unité de traitement signifie que l'Espagne n'a jamais adopté une loi concernant la navigation par les détroits internationaux, différente ou éloignée des règles régissant la navigation dans sa mer territoriale.

C'est ainsi que le régime de la navigation par les détroits, dont l'Espagne est riveraine, est régi par les règles commentées ci-dessus pour la mer territoriale, avec la seule exception que l'Etat espagnol a toujours appliqué pour ses détroits la règle internationale, d'origine coutumière, bien que déjà codifiée dans l'art. 16.4 de la Convention de 1958 sur la mer territoriale, qui veut

\footnotetext{
37 Ibidem, art. 80.

38 Ibidem, art. 74. La situation des navires de guerre nucléaires étrangers par rapport à cette disposition reste obscure, en ce qui conceme l'art. 69 in fine faisant exception à son application. Il n'est pas clairement défini s'ils sont exempts de tout le contenu de l'art. 74 ou seulement de la possibilité d'être inspectés, mais devant demander également l'autorisation préalable pour naviguer dans la mer territoriale espagnole. Cette demière solution est celle qui a été convenue dans l'art. 11.2 de la Convention du ler décembre 1988 entre le Royaume d'Espagne et les Etats-Unis d'Amérique sur la coopération pour la défense.

39 Voir supra note 13.

40 Pour une description de ces derniers, v. Doc. A/Conf.13/6 et add. 1 du 23 octobre 1957 (Kennedy), Brève étude géographique et hydrographique des détroits qui sont des routes de navigation internationale, in III CNUDM, Documentos oficiales, I, (documentos preparatorios), p. 124-125 et 146.
} 
que le passage inoffensif de navires étrangers à travers les détroits ne peut pas être suspendu.

$\mathrm{Au}$ cours des diverses sessions du Comité des Fonds Marins et de la III CNUDM, l'Espagne fut ainsi l'Etat qui plaida avec le plus de constance pour la règle voulant que le régime du passage inoffensif sans suspension s'applique aux détroits utilisés pour la navigation internationale. Cette défense du passage inoffensif sans suspension s'est vue completée par l'affirmation d'après laquelle dans le détroit de Gibraltar il n'existe pas de régime conventionnel de longue date qui permette la libre navigation, ${ }^{41}$ et, évidemment, par l'opposition à l'affirmation d'un droit de passage en transit.

A partir de la deuxième session de la Commision des Fonds Marins, la délégation espagnole définit les grands axes de sa conduite tout au long des débats de la Commission et, plus tard, de la III CNUDM. L'Espagne s'opposa, à plusieurs reprises, à la prétention des grandes puissances maritimes d'introduire la liberté de navigation et de survol dans les détroits utilisés pour la navigation internationale compris dans la mer territoriale d'un ou plusieurs Etats riverains. Selon l'Espagne, n'importe quelle concession sur ces thèmes n'aurait été utile qu'aux navires et aéronefs militaires des grandes puissances. L'importante modification du droit international en vigueur qui s'en serait suivie aurait pesé inutilement sur les Etats riverains de ces détroits. Ces Etats apparaissaient comme les victimes des exigences politiques, militaires et stratégiques des super-puissances. De l'avis de la délégation espagnole, le droit international en vigueur, aussi bien coutumier que conventionnel, imposait que la navigation à travers ces détroits se réalise sous le même régime que le passage inoffensif par la mer territoriale, avec la seule réserve que le passage à travers les détroits ne pouvait pas être suspendu. Le principe du passage inoffensif à travers les détroits exigeait que les sous-marins naviguent en surface et excluait que les aéronefs en jouissent. ${ }^{42}$ Pour soutenir sa thèse, la délégation espagnole parvint à constituer, coordonner et diriger un groupe "d'Etats riverains de détroits". Ces Etats concrétisèrent ces idées dans un projet d'articles portant sur la navigation

\footnotetext{
41 Au cours des débats de 1973 à la Commission des Fonds Marins, il faut souligner qu'autant les délégations d'Italie (19 juillet 1973) que'celle de l'Union Soviétique (24 juillet 1973) proposèrent un régime de libre navigation dans ce détroit, se basant surtout sur l'art. VII de la déclaration Franco-Britannique du 8 avril 1904, ce qui valut la riposte percutante des délégations du Maroc (12 août 1973), et de l'Espagne (19 et 25 juillet 1975), à ce moment-là riverains de ce détroit. Cfr. N.U., El Derecho del Mar. Estrechos utilizados para la navegación internacional. Historial Legislativa de la tercera parte de la Convención de las Naciones Unidas sobre el derecho del mar, 1992, I, p. 121 et ss. Antérieurement à ce débat, le Ministre des Affaires Etrangères espagnol, Sr. Gregorio López Bravo, avait déjà soutenu cette thèse, le 3 février 1971, en affirmant que: "Parmi tous les détroits internationaux qui ne jouissent pas d'un régime spécial, celui qui a la plus grande importance, de par le tonnage du trafic qui le traverse et de sa valeur stratégique, c'est celui de Gibraltar", reproduit dans DE YTURRIAGA BARBERAN, Textos... cit., supra note 1, II, 2, p. 397 et ss.

42 Voir les interventions de la délégation d'Espagne dans cette Commission, reproduites dans N.U., El Derecho del Mar. Estrechos... cit., supra note 41, p. 33-35, 48-49, 57, 72-73, 100-101, 123-124, $132-134$ et 147 .
} 
dans la mer territoriale, incluant les détroits utilisés pour la navigation internationale. 43

$\mathrm{Au}$ début de la III CNUDM, l'Espagne continua à maintenir ses positions antérieures. La pression des grandes puissances maritimes, ajoutée à l'indifférence généralisée de la plupart des Etats du tiers-monde, fit, toutefois, perdre progressivement sa cohésion interne au groupe des "Etats riverains de détroits".

D'autre part, afin d'obtenir un consensus généralisé sur la navigation internationale à travers les détroits, la délégation espagnole, tout au long de la III CNUDM, renonça à défendre plusieurs des points qui constituaient une bonne partie du contenu de sa thèse officielle.

La consécration définitive du passage en transit dans la Partie III de la Convention de Montego Bay détermina l'abstention de la délégation espagnole au moment du vote. Elle affirma que "les intérêts de son pays n'avaient pas été vraiment pris en compte", 44 ajoutant par ailleurs que "les textes approuvés par la Conférence ne constituent pas une codification ou une expression du droit coutumier", du moins en ce qui concerne la question des détroits. Cette attitude fut renforcée par les déclarations interprétatives formulées par l'Espagne le 4 décembre 1984 à la signature de la Convention de Montego Bay. 45

Suite à l'adoption de la Convention, l'attitude de l'Etat espagnol n'a pas changé par rapport à ce que doit être le régime de la navigation internationale à travers les détroits dont l'Espagne est un Etat riverain et, en particulier, en ce qui concerne le détroit de Gibraltar.

Ainsi, on n'a pas modifié la législation espagnole réglementant la navigation aérienne dans l'espace aérien espagnol au-dessus du territoire et de la mer territoriale, incluant le survol des détroits utilisés pour la navigation internationale. En conséquence, les art. 2.2 et 89 de la loi 48/1960 du 21 juillet concernant la navigation aérienne ${ }^{46}$ continuent à exiger l'autorisation préalable de l'Etat pour le passage des aéronefs étrangers dans l'espace aérien espagnol au-dessus de ses détroits.

En ce qui concerne la navigation maritime, les changements introduits dans la législation, après l'adoption de la Convention de Montego Bay, ont voulu renforcer la prétention selon laquelle à travers ces détroits internationaux, on ne peut naviguer qu'en régime de passage inoffensif. Si l'arrêté du Ministère de la Marine 885/1958 du 29 mars 47 affirmait "qu'il n'était pas demandé d'autorisation spéciale pour le transit à travers les eaux territoriales espagnoles" des navires de guerre étrangers, en revanche l'arrêté du Ministère de la Défense 25/1985 du 23 avril, établissant les normes por-

\footnotetext{
43 Projet d'articles présenté par Chypre, Espagne, Philippines, Grèce, Indonésie, Malaisie, Maroc et Yémen. Publié ibidem, p. 81-89.

44 Voir supra note 3.

45 Reproduites dans 4 Bull. D.M., 1985, p. 14-15 et 5 Bull. D.M., 1985, p. 21 et 42-43.

46 Voir supra note 23.

47 Voir supra note 30.
} 
tant sur les escales des navires de guerre étrangers et sur leur passage à travers la mer territoriale espagnole en temps de paix, ${ }^{48}$ et annulant l'arrêté de 1958, stipule à l'art. 11.1 que:

"Il n'est pas demandé d'autorisation spéciale pour le passage de navires de guerre étrangers à travers la mer territoriale espagnole, où ils sont dans l'obligation de respecter le passage inoffensif, conformément aux règles coutumières du droit international".

Il faut ajouter que le rapport accompagnant le projet de l'arrêté 25/1985, affirme que la suppression du terme "transit" "a pour but d'éviter de possibles confusions avec le "passage en transit" créé par la Convention des Nations Unies en relation avec le passage par les détroits". ${ }^{49}$ L'arrêté 25/1985 inclut également une affirmation catégorique de la part de l'Etat espagnol d'après laquelle "les normes coutumières du droit international" qui réglementent le passage à travers les détroits utilisés pour la navigation internationale sont celles qui prévoyent la navigation en régime de passage inoffensif sans possibilité de suspension.

\section{ZONE CONTIGUË, ZONE ARCHEOLOGIQUE, AUTRES ZONES FONCTIONNELLES NE PORTANT PAS SUR LES RESSOURCES ECONOMIQUES}

Quant à la zone contiguë, le décret 3.281/1968 du 26 décembre, modifia l'art. 33 de l'Ordonnance des Douanes comme suit:

“... l'action fiscale à effets douaniers, et visant la répression de la contrebande, s'exerce dans les eaux territoriales qui, aux effets fiscaux, comprennent une zone de mer adjacente aux côtes espagnoles de 12 milles de largeur". 50

Par la suite, l'extension de la mer territoriale à $12 \mathrm{~m} . \mathrm{m}$. fit perdre sa raison d'être à la zone contiguë, dans l'attente de son éventuelle extension aux 24 m.m. que le droit de la mer d'aujourd'hui permet. La réintroduction dans le droit espagnol d'une zone contiguë, qui atteint aujourd'hui 24 m.m., se fit avec la loi 27/1992 du 24 novembre sur les ports de l'Etat et la Marine Marchande (art. 7.1). La définition du régime juridique de la zone contiguë espagnole a été améliorée par rapport au décret de 1968, en établissant que:

\footnotetext{
48 Voir supra note 19.

49 Etat-Major de l'Armée, rapport justificatif s. d., p. 1.

50 B.O.E. du 20 janvier 1969.
} 
“... dans la zone contiguë, définie par l'article 7.1 de la présente loi, le Gouvernement pourra adopter les mesures fiscales nécessaires pour:

a) empêcher, sur le territoire national ou sur la mer territoriale, les infractions aux lois et aux règlements douaniers, de contrebande, fiscaux, d'immigration ou sanitaires;

b) sanctionner lesdites infractions". 51

Il n'existe aucune norme internationale qui établisse un régime de navigation différent pour la zone contiguë. Il s'ensuit que, dans la zone contiguë, valent les libertés de navigation et de survol prévues pour la haute mer. Les mesures fiscales que l'Etat espagnol peut et doit appliquer à sa zone contiguë pour prévenir et réprimer les infractions à ses lois et règlements douaniers, de contrebande, fiscaux, d'immigration ou sanitaires, 52 interfèrent ou peuvent interférer avec la liberté de navigation pour tous les navires étrangers et nationaux dans cet espace maritime espagnol. Ainsi, les art. 111 et 112 de la loi sur les ports de l'Etat et la Marine Marchande établissent que l'Etat espagnol a, dans sa zone contiguë, exactement les mêmes pouvoirs de police examinés plus haut à propos de la navigation dans la mer territoriale espagnole.

Il faut ajouter, enfin, que le droit espagnol n'a pas exploité toutes les potentialités de la zone contiguë, puisqu'il n'a pas établi, en conformité avec l'art. 303.2 de la Convention de Montego Bay, une zone archéologique de 24 m.m. comme certains Etats voisins l'ont fait. 53

\section{PECHERIES ET ZONE ECONOMIQUE EXCLUSIVE}

\subsection{Limites extérieures}

La législation espagnole au sujet de la zone de pêche a subi une évolution constante. Suite à la Convention européenne sur la pêche (Londres, 9 mars 1944) qui établissait un système de " $6+6$ ",54 on édicta la loi 20/1967 du 8 avril étendant les eaux territoriales espagnoles à $12 \mathrm{~m} . \mathrm{m}$. en ce qui concerne la pêche. ${ }^{55}$ Par la suite, deux accords de pêche furent conclus avec la

51 Disposition additionnelle deuxième de la loi 27/1992 du 24 novembre. Voir supra note 13.

52 Disposition additionnelle 2 de la loi 27/1992 du 24 novembre, des ports de l'Etat et de la Marine Marchande, op. cit.

53 Voir la loi française 89-874 du 11 décembre 1989, relative aux biens culturels maritimes et modificative de la loi du 27 septembre 1941 réglementant les excavations archéologiques, 16 Bull. D.M., 1990 , p. 13 et 55.

54 Non publié dans le B.O.E. Le texte de la Convention peut-être consulté dans SANCHEZ RODRIGUEZ, España y el régimen internacional de la pesca maritima, 1986, p. 117. Dans les 6 prémiers m.m., l'Etat riverain avait le droit et la juridiction exclusive en matière de pêche; entre les 6 et les 12 m.m., en plus de l'Etat riverain, pouvaient pêcher ceux qui avaient "pratiqué habituellement la pêche dans cette zone".

s5 Voir supra note 5. 
France et le Portugal. ${ }^{56} \mathrm{La}$ consécration internationale de la zone économique exclusive (ZEE) touche de façon préjudiciable l'Espagne en tant que grande puissance de pêche, avec des intérêts dans la pêche à distance, qu'elle était et qu'elle est. Lorsque la ZEE fut irrémédiablement consacrée par le droit international général, l'Espagne ne put faire autrement que créer sa propre ZEE et négocier un grand nombre de traités pour accéder à des lieux de pêche auparavant considérés comme parties de la haute mer.

La loi 15/1978 du 20 février ${ }^{57}$ créa la ZEE espagnole s'étendant jusqu'à une distance de $200 \mathrm{~m} . \mathrm{m}$. L'Etat espagnol y jouit de droits souverains en ce qui concerne l'exploration et l'exploitation des ressources naturelles du lit ainsi que du sous-sol marin et des eaux surjacentes (art. 1.1). Cette loi s'applique seulement aux espaces maritimes situés sur la façade Atlantique, la Mer Cantabrique comprise, autorisant le Gouvernement à procéder, ce qu'à ce jour il n'a pas encore fait, à son extension réglementaire à la Mer Méditerranée (disposition finale 1).

\subsection{Régime}

Moyennant la conclusion d'un accord préalable avec les gouvernements étrangers concernés, ${ }^{58}$ on a réglementé aussi bien les conditions de permis de pêche à obtenir par les navires étrangers pour opérer dans les eaux espagnoles, que les infractions administratives qui, en matière de pêche, sont commises dans les eaux se trouvant sous la juridiction espagnole ou celles commises par les navires à pavillon espagnol dans les eaux se trouvant sous la juridiction d'autres Etats et en haute mer. 59

Afin d'assurer la compétitivité du secteur de pêche espagnol, suite à l'institution généralisée des ZEE, l'Espagne a développé une vaste pratique conventionnelle, en participant à plusieurs accords de coopération de pêche dans des milieux géographiques limités, ${ }^{60}$ ainsi qu'à des accords sur les espèces protégées ou fortement migratrices. 61

56 Avec la France (Paris, 20 mars 1967), B.O.E. du 8 décembre 1970; avec le Portugal (Madrid, 9 décembre 1969), B.O.E. du 19 novembre 1977. Ce système fut même élargi aux relations de pêche bilatérales avec un Etat non Européen. Voir l'Accord sur la pêche maritime avec le Maroc (Fez, 4 janvier 1969), B.O.E. du 5 juin 1969.

57 Voir supra note 11.

58 Arrêté du 2 mars 1982, B.O.E. du 22 mars 1982.

59 Loi 53/1982 du 13 juillet au sujet des infractions administratives en matière de pêche maritime, B.O.E. du 30 juillet 1982 .

60 Mis à part la Convention européenne sur la pêche, voir supra note 54, l'Espagne participe à la Convention établissant le Conseil général des pêches pour la Méditérranée (Rome, 24 septembre 1949), B.O.E. du 4 août 1979; à la Convention sur la future coopération multilatérale dans la pêche de l'Atlantique du Nord-Ouest (Ottawa, 24 octobre 1978), B.O.E. du 27 septembre 1983; à la Convention sur la future coopération multilatérale dans la pêche de l'Atlantique du Nord-Est (Londres, 18 novembre 1980), B.O.E. du 3 septembre 1984; à la Convention sur la conservation de la faune et de la flore marines de l'Antarctique (Camberra, 20 mai 1980), B.O.E. du 25 mai 1985.

61 Convention sur la conservation des thonidés atlantiques (Rio de Janeiro, 14 mai 1966), B.O.E. du 22 avril 1969; Convention pour la réglementation de la chasse à la baleine (Washington, 2 décembre 1946), B.O.E. du 24 mai 1985. 
Dans les nombreux accords bilatéraux de pêche que l'Espagne a conclu avec des pays européens, ${ }^{62}$ africains ${ }^{63}$ et américains, ${ }^{64}$ ont été également réglementées les conditions pour l'obtention du permis temporaire de pêche par les navires espagnols qui oeuvrent dans les eaux territoriales des autres Etats, ou en haute mer. ${ }^{65}$ Toute cette pratique conventionnelle s'est vue complétée par la création de nombreuses entreprises de pêche conjointes, qui sont définies comme celles qui:

“... dans un pays étranger, et conformément à sa législation, constituent les entreprises de pêche espagnoles en association avec des personnes physiques ou juridiques dudit pays et, le cas échéant, d'autres pays, dans le but d'exploiter conjointement les ressources de la pêche maritime". 66

L'adhésion de l'Espagne à la Communauté européenne porta un changement complet à cette situation. La Communauté européenne, à cause des intérêts contradictoires de différents Etats membres, ne parvint pas à établir une politique commune sur ce thème jusqu'en 1983 et, même à ce moment, celle-ci ne s'appliqua pas à la Mer Méditerranée. 67 Etant donné que les navires de pêche espagnols représentaient plus de $30 \%$ de la totalité de la flotte communautaire avec un tonnage supérieur à $60 \%$ de celle-ci, il ne faut pas s'étonner que l'acte d'adhésion (chapitre 4 du titre II) contienne des dispositions expresses à ce sujet.

Une longue période de transition fut établie (jusqu'au 31 décembre de l'an 2002) pour la pleine intégration de l'Espagne dans la politique de

\footnotetext{
62 Mis à part les accords avec la France et le Portugal, cit. supra note 56, v. les accords avec le Portugal (Lisbonne, 22 septembre 1978), B.O.E. du 30 janvier 1979; avec la Communauté européenne (Bruxelles, 15 avril 1980), B.O.E. du 25 mars 1982; avec la Norvège (Oslo, 21 janvier 1981), B.O.E. du 24 novembre 1982.

63 Accords avec le Maroc (Rabat, 1er août 1983), B.O.E. du 11 octobre 1983 (il faut signaler que les relations conventionnelles de pêche avec le Maroc remontent à 1969); avec l'Afrique du Sud (Prétoria, 14 août 1979), B.O.E. du 4 juin 1982; avec la Guinée équatoriale (Madrid, 31 octobre 1979), B.O.E. du 13 mars 1980; avec le Cap Vert (Madrid, 25 septembre 1981), B.O.E. du 28 novembre 1981; avec le Mozambique (Maputo, 19 décembre 1981), B.O.E. du 18 mai 1982; avec la Mauritanie (Madrid, 6 avril 1982), B.O.E. du 24 mai 1982, pays avec lesquels des relations conventionnelles sont maintenues sur ce thème depuis 1964; avec les Seychelles (Victoria, 1 juin 1984), B.O.E. du 4 septembre 1985; avec l'Angola (Luanda, 2 novembre 1984), B.O.E. du 4 septembre 1985; avec la Guinée (Conakry) (Madrid, 9 octobre 1984), non publié dans le B.O.E. (le texte de l'Accord peut-être consulté dans le B.O.C.G., Congreso, II Leg., Serie C, du 29 juillet 1985); avec le Sénégal (Madrid, ler mars 1985), B.O.E. du 30 août 1985; correction des erreurs dans le B.O.E. du 24 octobre 1985.

64 Accords avec le Canada (Madrid, 10 juin 1976), B.O.E. du 5 novembre 1976; avec les Etats-Unis (Washington, 29 juillet 1982), B.O.E. du 17 novembre 1983; correction des erreurs dans B.O.E. du 21 avril 1983.

65 Arrêté du 2 mars 1982, B.O.E. du 23 mars 1982.

66 Décret royal 830/1985 du 30 avril, sur les entreprises de pêche unies, B.O.E. du 6 juin 1985. Le recours à la constitution de ce type d'entreprises fut fréquent en Amérique centrale et Amérique du sud, ce qui explique l'inexistence d'accords de pêche bilatéraux avec le pays de ce continent.

67 Voir le par. 1 du préambule du règlement du Conseil (CEE) n. 170/83 du 25 janvier 1983, établissant un régime communautaire de conservation et de gestion des ressources de la pêche JOCE L $24 \mathrm{du}$ 27 janvier 1983.
} 
pêche communautaire. ${ }^{68}$ Durant cette période transitoire, l'accès des navires de pêche espagnols aux eaux et aux ressources communautaires dépend du nombre de navires autorisés à pêcher dans les eaux espagnoles ainsi qu'aux quotas assignés sur le total admissible de captures (TAC). ${ }^{69}$ Des régimes spéciaux de pêche furent créés pour les Iles Canaries et Ceuta et Melilla, ${ }^{70}$ de même que pour la pêche espagnole dans les eaux portugaises et vice-versa. ${ }^{71} \mathrm{D}$ 'autre part, l'accès à la ZEE espagnole des navires de pêche des autres Etats membres, ${ }^{72}$ dépend des institutions communautaires. Quant aux accords avec les Etats tiers

“... dès le moment de l'adhésion, la gestion des accords de pêche conclus par le Royaume d'Espagne avec des Etats tiers sera menée par la Communauté".

Lorsque l'accord prend fin, sa renégociation ${ }^{73}$ incombe également à la Communauté. C'est ainsi que, depuis son adhésion, l'Espagne n'a plus conclu d'accords de ce type. ${ }^{74}$ Enfin, il convient de signaler qu'il fut établi une période de sept ans pour arriver à l'élimination totale de toute sorte d'exonération, suspension ou contingent douanier ${ }^{75}$ pour les entreprises de pêche conjointes constituées par l'Espagne et les Etats tiers.

A cause de la dureté des conditions imposées à l'Espagne par l'acte d'adhésion en matière de pêche, il ne faut pas s'étonner qu'après l'adhésion, l'Etat espagnol ait voulu améliorer par tous les moyens possibles sa situation dans ce domaine. Outre les négociations annuelles où l'Espagne a tenté d'augmenter ses possibilités de pêche dans les eaux communautaires, ${ }^{76}$ il convient de rappeler qu'un des axes fondamentaux de la politique

68 Ibidem, art. 166.

69 Ibidem, art. 157 à 161 .

70 Ibidem, art. 155.

71 Ibidem, art. 165 et 352.

72 Ibidem, art. 164.

73 Ibidem, art. 167. Cela a déjà commencé à arriver, par exemple, avec les accords de pêche que l'Espagne avait conclu avec le Maroc (v. JOCE L 181 du 12 juillet 1988) ou avec les Seychelles (v. B.O.C.G., Congreso III Leg., serie C, du 28 mars 1987). Cependant, dans quelques cas, comme dans les relations de pêche avec le Maroc, cette substitution dans la négociation augmenta le nombre de captures des navires espagnols qui oeuvrent dans ces eaux. Voir B.O.C.G., Congreso, IV Leg., Diario de Sesiones, Comisiones, n. 93, 29 mai 1990, p. 2755.

74 Il convient toutefois de faire référence à un type d'accord bilatéral atypique, bien que prévu à l'art. 5 du règlement du Conseil (CEE) n. 170/83 du 25 janvier 1983, établissant un régime communautaire de conservation et de gestion des ressources de la pêche (JOCE L 24 du 27 janvier 1983), et à l'art. 9 du règlement du Conseil (CEE) n. 3760/92 du 20 décembre 1992, établissant un régime de pêche et acquaculture (JOCE L 389 du 31 décembre 1992). En application du mandat du Conseil des Ministres de la pêche des Communautés européennes pour rationnaliser la pêche de l'anchois dans le lieu de pêche du Cantabrique, le Ministère espagnol de l'Agriculture, de la Pêche et de l'Alimentation, a conclu à partir de 1992, des traités bilatéraux avec le Ministère Français de l'Agriculture et de la Pêche relatifs à l'échange de quotas d'anchois avec d'autres productions de la pêche, réglementant aussi des périodes de fermeture et d'inactivité dans ce secteur.

75 Ibidem, art. 168.

76 Ibidem, art. 162. 
de pêche espagnole en matière de ressources a toujours été d'assurer et même d'augmenter les possibilités de pêche pour les navires espagnols dans les eaux des Etats tiers. ${ }^{77}$

D'autre part, le Conseil des Ministres qui a eu lieu à Luxembourg les 17 et 18 juin 1991, adopta un règlement autorisant l'intégration totale des Canaries dans plusieurs politiques communautaires, y compris la politique de pêche commune. ${ }^{78} \mathrm{D}$ 'après ce règlement, l'incorporation des Canaries à la politique de pêche commune se produit selon les mêmes modalités applicables au reste du secteur de pêche espagnol.

Mais la plus grande réussite espagnole est probablement celle d'avoir obtenu d'avancer de six ans l'intégration complète de l'Espagne dans la politique de pêche commune. Avec le prétexte des négociations d'adhésion à l'Union européenne de quatre nouveaux Etats, et en particulier de la Norvège, l'Espagne a ainsi adopté une politique de force consistant à prétendre obtenir 14.000 tonnes de morue dans les eaux norvégiennes. Bien que le quota finalement obtenu fût bien moins important, il fut convenu au nombre d'autres mesures ${ }^{79}$ que l'intégration complète de l'Espagne et du Portugal dans la politique de pêche commune devienne effective à partir du ler janvier 1996.80 Toutes les forces politiques représentées au Congrès des

77 En réponse à une question formulée au Congrès des Députés, le Ministre de l'Agriculture, de la Pêche et de l'Alimentation, M. Atienza Serna, affirma la chose suivante: 'L'Union Européenne a actuellement 23 accords de pêche en vigueur et dans une bonne partie de celle-ci, en particulier les accords avec les pays africains, l'Espagne bénéficie de façon majoritaire des possibilités de pêche inclues dans ces accords. Dans ce sens, je veux rappeler à ces Messieurs que le 4 mai passé, l'une des plus importantes demandes espagnoles fut honorée, avec l'entrée en vigueur de l'Accord de pêche de la Communauté avec l'Argentine, premier accord de la deuxième génération que la Communauté européenne signe et qui ouvre un nouvel horizon de perspectives pour la flotte frigorifique espagnole. Les armateurs espagnols ont déjà présenté vingt projets de societés mixtes et trois projets d'associations temporelles d'entreprises. Je veux aussi rappeler à ces Messieurs que l'une des conséquences de l'entrée en vigueur, le ler janvier 1994, des prévisions contenues dans l'Accord sur l'Espace Commun Européen et étant donné la position espagnole, fermement maintenue durant ces négociations, notre flotte morutière, a pu oeuvrer à partir de cette année et pour la première fois depuis 1980, dans les eaux territoriales de la Norvège. De la même manière, et dans l'attente que l'incorporation des quatre nouveaux Etats membres à l'Union européenne se produise, une nouvelle amélioration des possibilités de pêche pour la flotte morutière espagnole a été obtenue dans le processus de négociation de son adhésion, de même que quelques autres avantages additionnels", B.O.C.G., Diario de Sesiones del Congreso de Diputadoss, Pleno y Diputación Permanente, V Leg., 14 septembre 1994, p. 4566-4567.

78 Voir la comparution du Secrétaire d'Etat pour les Communautés européennes, D. Carlos Westendorp y Cabeza, devant la Commission des Affaires Etrangères du Congrès des Députés pour rendre compte sur "les Canaries: nouveau régime spécifique d'intégration à la CEE", B.O.C.G., Diario de sesiones del Congreso de los Diputados, comisiones 26 septembre 1991.

79 L'Espagne obtint finalement l'accès à 6.000 tonnes de morue: de celles-ci, 1.250 tonnes provienraient des eaux norvègiennes, le reste serait négocié à Svalbard ou avec la Russie.

80 Le Conseil des Ministres des Affaires Générales du 16 mars 1994 adopta un important accord politique concrétisé dans une déclaration commune du Conseil et de la Commission, dont le deuxième paragraphe dit: "à cette fin, le Conseil apportera avant le ler janvier 1995, les mesures relatives aux conditions d'accès aux eaux et aux ressources envisagées dans l'Acte d'adhésion de l'Espagne et du Portugal, applicables à tous les navires communautaires. Ces mesures entreront en vigueur au plus tard le ler janvier 1996, afin de résoudre les problèmes spécifiques de l'Espagne et du Portugal". Cet accord se matérialisa avec la publication dans le JOCE L 140 du 3 juin 1994, du règlement de fixations correspondant. 
Deputés étaient par ailleurs unanimes sur la nécessité d'associer l'élargissement de l'Union européenne avec la pleine intégration de l'Espagne dans la politique de pêche communautaire. ${ }^{81}$

Finalement, il faut signaler que restent à l'Espagne, de même qu'aux autres Etats membres, des compétences résiduelles dans le domaine de la pêche, étant donné que l'art. 10.1 du règlement du Conseil (CEE) n. 3760/92 du 20 décembre 1992, par lequel est établi un régime communautaire de la pêche et de l'aquaculture, ${ }^{82}$ contient les dispositions suivantes:

"Les Etats membres peuvent prendre des mesures de conservation et de gestion des ressources dans les eaux sous leur souveraineté ou leur juridiction, pour autant:

- qu'elles concernent les stocks strictement locaux ne présentant un intérêt que pour les pêcheurs de l'Etat membre concerné;

- qu'elles s'appliquent uniquement aux pêcheurs de l'Etat membre concerné;

- qu'elles soient compatibles avec les objectifs visés à l'article 2 paragraphes 1 et 2 et ne soient pas moins rigoureuses que les mesures adoptées conformément à l'article 4".

\subsection{Navigation dans la zone économique exclusive}

En anticipant sur l'art. 58.1 de la Convention de Montego Bay, selon lequel tous les Etats jouissent, dans la ZEE, des libertés de navigation et de survol propres à la haute mer, l'art. 5.1 de la loi $15 / 1978$ du 20 février, instituant la ZEE espagnole, ${ }^{83}$ affirme que la mise en place de cet espace maritime espagnol n'affecte pas les libertés de navigation et de survol. Dès lors, il n'existe pas dans le droit espagnol une réglementation détaillée de la navigation aérienne ou maritime concernant sa ZEE, bien que des aspects très

\footnotetext{
81 Voir le débat sur la motion du groupe Izquierda-Unida Initiativa per Catalunya, subséquent à l'interpellation urgente au sujet de la politique du Gouvernement, pour garantir, fortifier et développer le secteur de pêche national, dans laquelle on pria le gouvemement d'agir avec la plus grande fermeté dans l'Union européenne, demandant que les droits des pêcheurs espagnols soient exactement les mêmes que ceux de n'importe quel autre pêcheur d'une autre nation dans le domaine de l'Union européenne. En B.O.C.G., Diario de sesiones del Congreso de los Disputados, Pleno y Diputación Permanente, V Leg., 20 septembre 1994, p. 4668 et ss. Voir également le débat sur trois propositions qui ne sont pas de loi, soumises au Congrès par le Groupe Parlamentario Popular, Socialista e Izquierda Unida Initiativa per Catalunya, qui furent revues dans un texte transactionnel au contenu suivant: "Le Congrès des Députés demande au gouvernement qu'il présente l'instrument de ratification du Traité d'Adhésion à l'Union européenne de l'Autriche, de la Finlande, de la Norvège et de la Suède après les engagements assumés par l'Union européenne dans les négociations pour garantir l'équilibre de celle-ci, et en particulier après que le Conseil des Ministres de l'Union ait adopté les règlements, ou accords précis, par lesquels la totale intégration de l'Espagne au régime général de la politique commune de pêche se fasse effective, et prenne effet à partir du ler janvier 1996", ibidem, 4 octobre 1994, p. 4882 et ss.

82 Voir supra note 74.

83 Voir supra note 11.
} 
concrets en relation avec celle-ci aient été réglementés sur la base de la Convention de Montego Bay.

Ainsi est-il que les navires de pêche étrangers, lorsqu'ils exercent leur droit de libre navigation à travers la ZEE espagnole, sont tenus de respecter les dispositions espagnoles destinées à empêcher qu'ils se consacrent à la pêche dans ce secteur maritime, y compris les dispositions concernant l'arrimage des engins de pêche. ${ }^{84}$ Cette prescription trouve son fondement dans l'art. 58.2 de la Convention de Montego Bay, par lequel la liberté de navigation en haute mer s'applique à la ZEE dans la mesure où celle-ci n'est pas incompatible avec les dispositions qui réglementent ce secteur maritime.

D'autres restrictions à la liberté de navigation dans la ZEE espagnole, impensables en haute mer, peuvent surgir lorsque l'exercice de celle-ci met, ou peut mettre, en danger les droits reconnus à l'Espagne en tant qu'Etat riverain. Une application de l'art. 58.3 de la Convention de Montego Bay est contenue dans l'art. 112 de la loi 27/1992 du 24 novembre, sur les ports de l'Etat et la Marine Marchande 85 qui, résumant l'art. 73.1 de la Convention de Montego Bay, étend à ce secteur maritime les pouvoirs de police de l'Etat espagnol. Ainsi, afin de sauvegarder la sécurité de la navigation et d'empêcher la pollution du milieu marin, l'Administration espagnole pourra, dans la ZEE, visiter, inspecter, conditionner le mouillage, arraisonner, entamer une procédure judiciaire et, de manière générale, adopter les mesures estimées nécessaires à l'égard des navires qui portent atteinte ou peuvent porter atteinte à ses droits.

Beaucoup plus incertaine semble, à première vue, la compatibilité avec la Convention de Montego Bay de l'arrêté du 17 avril 1991, réglementant le mouillage des navires-citernes dans les eaux territoriales ou dans la ZEE espagnole. ${ }^{86}$ Soumettre le mouillage des navires pétroliers dans la ZEE espagnole à l'autorisation préalable de l'Administration maritime espagnole s'accorde mal avec la liberté de navigation propre à la haute mer. Toutefois, la finalité de cet arrêté ministériel (sauvegarder le trafic maritime, les ressources naturelles et environnementales des zones maritimes touchées ou qui peuvent l'être dans l'avenir) met en évidence qu'il s'agit de matières sur lesquelles l'Etat espagnol a des droits de souveraineté ou de juridiction, conformément à l'art. 56 de la Convention de Montego Bay. Ainsi, on peut considérer que la solution adoptée par l'arrêté commenté n'est pas contraire au droit international. Elle représente un équilibre raisonnable entre les compétences de l'Etat espagnol sur sa ZEE et la liberté de navigation reconnue aux Etats tiers. De ce fait, l'arrêté est respecté sans qu'aucun Etat tiers ne le conteste.

\footnotetext{
84 Ibidem, art. 5.2.

85 Voir supra note 13.

86 Voir supra note 28.
} 
L'Accord du 26 octobre 1990, conclu entre l'Espagne et l'Union des Républiques Socialistes Soviétiques, concernant la prévention d'incidents dans la mer en dehors de la mer territoriale, 87 est beaucoup plus discutable. Dans le but de garantir la sécurité de la navigation maritime et aérienne des navires et aéronefs de leurs Forces Armées respectives en dehors de la mer territoriale, et étant entendu que les actes dangereux interdits dans cet accord ne doivent pas non plus être réalisés en ce qui concerne les navires civils qui arborent le pavillon de chacune des parties, les deux Etats décidèrent que:

"Les parties prendront des mesures pour former les commandants de leurs navires afin qu'ils observent strictement le contenu et l'esprit du Règlement International contre les abordages en mer de 1972. Les deux parties reconnaissent que le fondement de la liberté de navigation, et celui de mener à bien les opérations en dehors de la mer territoriale, se base sur les principes et les normes généralement acceptées du droit international, en particulier, les dispositions de la Convention de Genève concernant la haute mer, en date du 29 avril 1958". 88

Cet Accord suppose, entre autre, que l'Etat espagnol s'est rallié à la thèse maintenue par les grandes puissances, à savoir que les manoeuvres militaires dans la ZEE sont permises par le régime de libre navigation existant dans celle-ci. Dans tous les cas, sa conformité avec la Convention de Montego Bay est incertaine, quel que soit l'Etat riverain de la ZEE où se déroulent ces exercises militaires. Si ces exercices ont lieu dans la ZEE de l'une des parties participant à l'Accord, l'art. 58 de la Convention de Montego Bay serait applicable; s'ils ont lieu dans la ZEE d'un Etat tiers, l'art. 59 de la Convention de Montego Bay demande à ce que l'éventuel conflit d'intérêts soit résolu parmi tous les Etats impliqués, y compris l'Etat riverain,

“... sur la base de l'équité et eu égard à toutes les circonstances pertinentes, compte tenu de l'importance que les intérêts en cause présentent pour les différentes parties et pour la communauté internationale dans son ensemble".

\section{PLATEAU CONTINENTAL}

L'étendue géographique du plateau continental espagnol est assez réduite. En effet, si l'on considère la ligne isobathe des $200 \mathrm{~m}$, sa largeur ne dépasse pas les 15 m.m., excepté dans l'anse Gaditaine (15-30 m.m.) et dans les

87 B.O.E. du 4 novembre 1991.

88 Ibidem, art. 2. 
côtes Levantines (30-50 m.m.). La législation espagnole au sujet de cet espace maritime est incomplète et partielle car elle s'occupe de ressources très concrètes qui sont aussi bien des ressources non vivantes, ${ }^{89}$ que des espèces sédentaires. ${ }^{90}$ Pour combler cette lacune, il faut rappeler que l'Espagne adhéra en 1971 à la Convention de 1958 sur le plateau continental et que la loi 15/1978 du 20 février établit une ZEE de 200 m.m. dans l'Océan Atlantique, y compris la Mer Cantabrique. Par conséquent, le régime juridique du plateau continental espagnol se trouve, en règle générale, dans la Convention de 1958 sur le plateau continental.

Par ailleurs, la limite extérieure du plateau continental espagnol se trouvant dans l'Océan Atlantique, et incluant la Mer Cantabrique, est de 200 m.m., étant donné que le plateau continental est inclus dans la ZEE espagnole. Quant au plateau continental espagnol se trouvant dans la Mer Meditérranée, il existe encore en tant qu'espace autonome, étant donné que dans cette région maritime il n'y a pas de ZEE espagnole. Le plateau continental ne peut pas y avoir une limite extérieure allant jusqu'à $200 \mathrm{~m}$.m. car la géographie méditérranéenne le rend impossible. Par conséquent, sa limite extérieure sera donnée par les délimitations convenues avec ces Etats méditérranéens dont les plateaux continentaux sont contigus ou font face au plateau continental espagnol.

\section{LA HAUTE MER}

\subsection{Conditions requises pour battre pavillon}

L'Espagne n'est pas un Etat partie à la Convention des Nations Unies sur les conditions d'immatriculation des navires (Genève, 7 février 1986), ni de l'Accord plus récent de la FAO, visant à favoriser le respect, par les navires de pêche en haute mer, des mesures internationales de conservation et de gestion (Rome, 24 novembre 1993). Cependant, tant l'art. 5 de la Convention de 1958 sur la haute mer, ${ }^{91}$ que le droit international coutumier, obligent l'Etat espagnol à établir les formalités nécessaires pour octroyer sa nationalité aux navires, ainsi que pour leur immatriculation sur son territoire et pour qu'ils aient le droit d'arborer son pavillon.

Une profonde réforme des normes administratives espagnoles qui réglementent l'octroi de la nationalité, l'immatriculation et le registre des navires s'est produite au cours des dernières années. A l'origine de cette réforme on

\footnotetext{
89 Loi 22/1988 du 28 juillet, sur la protection, utilisation et police des côtes (B.O.E. du 29 juillet 1988) qui annula la précédente loi 28/1969 du 26 avril (B.O.E. du 28 avril 1969); loi 21/1974 du 27 juin sur les investigations et exploitation des hydrocarbures (B.O.E. du 29 juin 1974; correction des erreurs dans le B.O.E. du 6 août 1974); loi 22/1973 du 21 juillet, des mines (B.O.E. du 24 juillet 1973).

90 Loi 59/1969 du 30 juin, sur l'ordonnance mareyeuse (B.O.E. du ler juillet 1969); décret royal $1212 / 1984$ du 8 juillet, par lequel on réglemente la pêche du corail (B.O.E. du 26 juin 1984).

91 Voir supra note 2.
} 
trouve la dispersion normative qui existait, les exigences survenues comme conséquence de l'entrée de l'Espagne dans la Communauté européenne et le grand dynamisme du secteur maritime.

Cette réforme a été réalisée par le décret royal 1027/1989 du 28 juillet, sur la nationalité, l'immatriculation et le registre des navires ${ }^{92}$ et par la loi 27/1992 du 24 novembre sur les ports de l'Etat et la Marine Marchande. ${ }^{93}$ Cette loi a libéralisé la législation espagnole en soumettant celle-ci aux exigences de l'Acte Unique Européen et de la réalisation effective du Marché Unique Intérieur. ${ }^{94}$

\subsection{Pêche en haute mer}

L'Espagne est un Etat partie à la Convention de 1958 sur la haute mer ainsi qu'à la Convention de 1958 sur la pêche. ${ }^{95}$ La liberté de pêche en haute mer des navires de pêche espagnols n'en est pas moins limitée, car l'Espagne est partie aussi à plusieurs accords de coopération de pêche dans des domaines géographiques limités, qui comprennent parties de la haute mer, ${ }^{96}$ ainsi qu'à des accords sur des espèces protégées ou hautement migratrices. 97

Les limitations les plus importantes à l'exercice de la liberté de pêche en haute mer par les navires de pêche espagnols proviennent de l'adhésion de l'Espagne aux Communautés européennes en 1986. Dans le domaine de la politique extérieure de la pêche, la Communauté européenne prend part à plusieurs accords multilatéraux de pêche. Ce sont les institutions communautaires et non les Etats membres qui négocient dans les conférences internationales de pêche en haute mer.

Plus important encore est peut-être le processus, survenu au cours des dernières années, qui voit l'extension à la haute mer de la politique commune de pêche de l'Union européenne. L'Espagne n'a pas été étrangère à ce processus. Ainsi, il convient de faire référence à l'arrêt de la Cour de Justice des Communautés européennes du 25 juillet 1991, suite à une action introduite par la Commission contre l'Espagne. ${ }^{98} \mathrm{~A}$ l'origine de cette affaire on trouve des inspections réalisées par la Commission en 1986 et 1987. Ces inspections mirent en évidence qu'au cours de ces années l'Administration espagnole n'avait pas enregistré les captures d'espèces soumises à TAC ou aux quotas, qui avaient été pêchées dans certains sec-

\footnotetext{
92 B.O.E. du 15 août 1989.

93 Voir supra note 13; cette loi est dépourvue d'un développement reglementaire dans ce domaine.

94 D'aprés son préambule, cette loi établit les conditions pour que les navires puissent être enregistrés en Espagne et obtenir ainsi la nationalité espagnole, favorisant le principe libéral de la résidence ou du domicile des sujets titulaires, indépendemment de la nationalité.

95 Voir supra note 2.

96 Voir supra note 60.

97 Voir supra note 61.

98 Affaire C/258/89; Commission des Communautés européennes c. le Royaume d'Espagne, Recopilación de la juriprudencia del Tribunal 1991, p. 3977 et ss..
} 
teurs de pêche de l'Atlantique nord-oriental, sous le contrôle du Conseil International pour l'Exploration de la Mer (CIEM), en dehors de la limite des $200 \mathrm{~m} . \mathrm{m}$. de la zone de pêche de la Communauté. D'autre part, les inspections notèrent des débarquements illégaux de certaines espèces, déclarées pêchées dans l'ensemble des secteurs CIEM VI et VII, mais en dehors des 200 m.m., alors qu'il se trouvait que l'Espagne ne possédait pas de quotas de telles espèces dans les zones mentionnées. Les quatre inspecteurs constatèrent également que les autorités espagnoles n'avaient pas entamé d'actions pénales ou administratives à cet égard. ${ }^{99}$

Sur la base de ces faits, deux thèses juridiques distinctes s'affrontèrent. D'après la Commission, la compétence communautaire en matière de pêche présente deux aspects: ratione materiae, elle s'étend aux ressources biologiques de la mer dans les eaux sous la souveraineté ou la juridiction des Etats membres, et recouvre toutes les activités de pêche dans ces eaux; ratione personae, la compétence s'étend à toutes les activités de pêche des pêcheurs et navires des Etats membres, aussi bien dans la zone de pêche de la Communauté qu'en haute mer, et même à l'intérieur des zones de pêche des pays tiers. La Commission soutenait en outre que, dans ces deux derniers cas, la compétence de la Communauté s'exerce conformément aux normes du droit international. 100

L'Espagne opposa trois arguments différents. Primo, elle défendit la thèse que la pêche en haute mer, en dehors des restrictions convenues dans les accords internationaux spécifiques, est une activité libre et que, par conséquent, les quotas communautaires ne sont pas applicables dans les parties des divisions CIEM situées au-delà de la limite de la zone de pêche communautaire de 200 m.m. ${ }^{101}$ Secundo, le Gouvernement espagnol contesta le fait que la limitation autonome de l'effort de pêche applicable aux navires communautaires au-delà de sa zone de pêche soit un instrument utile pour obtenir l'objectif d'une conservation des ressources de pêche, étant donné que si la Communauté établissait des limitations autonomes, alors que les flottes des pays tiers agissaient librement, on ne favoriserait pas la conservation de la richesse halieutique mais on porterait plutôt préjudice aux navires communautaires au bénéfice des navires de pêche des pays tiers. ${ }^{102}$ Le Gouvernement espagnol précisa enfin que l'Espagne avait négocié l'accès aux eaux communautaires, mais non l'accès aux eaux internationales. Il ressortait de l'art. 156 de l'acte d'adhésion que la flotte espagnole avait libre accès aux eaux libres et aux ressources qui s'y trouvent. En effet, dans l'art. 161 de l'acte d'adhésion il n'est question que des eaux communautaires. ${ }^{103}$

\footnotetext{
99 Information pour la séssion présentée dans le cas C/258/89 point 3, ibidem, p. 3979.

100 Ibidem, point 9, p. 3981 et ss.

101 Ibidem, point 3, p. 3979.

102 Ibidem, point 20, p. 3985.

103 Ibidem, point 19, p. 3985.
} 
Il n'en reste pas moins que les conclusions de l'Avocat Général M. M. Darmon, ainsi que l'arrêt de la Cour de Justice, avec des prononcés clairement pro communitate, refusèrent une à une les diverses argumentations espagnoles. La Cour refusa la première allégation espagnole, affirmant que:

“... la Communauté a compétence pour prendre des mesures de conservation, et cela tant de manière autonome que sous la forme d'engagements contractuels avec des Etats tiers ou dans le cadre des organisations internationales...". 104

La deuxième allégation espagnole voulait prendre une avance dans le temps sur le traitement international des espèces chevauchantes (straddling stocks). ${ }^{105}$ Cette prétention fut également rejetée par la Cour, en affirmant que, d'une part, la seule prise en compte de la partie du stock qui se trouve à l'intérieur des eaux communautaires ne serait pas efficace et mettrait en danger les objectifs de conservation des espèces en cause, vu que ces dernières ne seraient plus soumises à quotas au moment où elles franchiraient la limite de la zone des $200 \mathrm{~m} . \mathrm{m}$. D'autre part, les captures effectuées dans la zone communautaire pourraient être facilement déclarées comme ayant été effectuées en haute mer. Il en découle que, dans n'importe quelle hypothèse, la limitation des possibilités de pêche en dehors de la zone communautaire est indispensable en fonction des objectifs de la politique commune de pêche. ${ }^{106}$

Enfin, l'argumentation selon laquelle l'on peut invoquer l'acte d'adhésion de l'Espagne et du Portugal pour contester la compétence de la Communauté à réglementer les possibilités de pêche espagnole en dehors des eaux communautaires, fut rejetée par la Cour de Justice par le bias d'une interprétation différente de l'acte d'adhésion. ${ }^{107}$

Pour toutes ces raisons, la Cour déclara que l'Espagne n'avait pas respecté les obligations lui incombant en vertu du Traité CEE, car elle n'a-

\footnotetext{
${ }^{104}$ Arrêt de la Cour de Justice du 25 juillet 1991, ibidem, par. 9, p. 4003.

105 La conférence sur le straddling stocks et les espèces fortement migratrices célébra sa première réunion organisée à New York du 19 au 23 avril 1993. Il faut signaler que, ni dans les allégations des parties, ni dans les conclusions de l'Avocat Général, ni dans le texte de l'arrêt, il n'est fait référence à l'art. 63 de la Convention de Montego Bay, concemant les stocks qui se trouvent à l'intérieur de ces ZEE de deux ou plusieurs Etats riverains, ou aussi bien à l'intérieur de la ZEE que dans une aire plus éloignée de celle-ci et adjacente à celle-ci.

106 Ibidem, par. 12 et 13, p. 4004.

107 Il faut prendre en compte que, d'après l'art. 156 de l'acte d'adhésion, l'accès de l'Espagne aux eaux soumises à la juridiction des Etats membres de la Communauté des dix est soumise à la section II du chapitre 4 du titre II de la quatrième partie du dit acte. Donc, dans cette section, les art. 157 à 160 établissent des normes relatives à l'accès à ces eaux, dès lors que l'art. 161 établit des quotas pour l'Espagne dans les zones VIII et IX, qui sont extérieures dans leur totalité aux eaux des Etats de la Communauté des dix. Etant donné que la répartition des ressources en rapport avec les zones extérieures aux eaux des Etats de la Communauté des dix est reglementée, il n'est pas utile d'invoquer le domaine d'application du régime d'accès aux eaux en ce qui conceme la répartition des quotas. Ibidem, par. 16, p. 4005 .
} 
vait pas appliqué aux captures de stocks, ou de groupes de stocks, soumises à un TAC ou à un quota, effectuées en dehors de la zone de pêche de la Communauté, les mesures de contrôle établies dans les règlements du Conseil (CEE) n. 2057/82 du 29 juin 1982 et n. 2241/87 du 23 juillet 1987, respectivement. 108

Il faut noter aussi que l'argumentation utilisée par la Commission pour défendre la politique commune de pêche en haute mer, fut reprise, à peine un an et demi plus tard, dans l'art. 1 du règlement du Conseil (CEE) $n$. 3760 du 20 décembre 1992, établissant un régime communautaire de la pêche et de l'aquaculture:109

"La politique commune de la pêche couvre les activités d'exploitation portant sur les ressources aquatiques vivantes et l'aquaculture, ainsi que la transformation et la commercialisation des produits de la pêche et de l'aquaculture, pour autant qu'elles soient pratiquées sur le territoire des Etats membres ou dans la zone de pêche communautaire ou par des navires de pêche communautaires".

\subsection{La pêche aux filets dérivants}

L'Espagne a été l'un des pionniers dans l'opposition à la pêche avec filets dérivants. L'arrêté ministériel du 24 novembre 1981,110 réglementa aussi bien l'utilisation des engins mineurs fixes, que celle des filets dérivants. Cet arrêté fut édicté exclusivement pour la Mer Méditérraneé étant donné qu'à cette date, c'était dans cette mer semi-fermée que l'incidence de l'utilisation de ces filets sur les ressources de pêche était la plus flagrante.

Presque dix ans plus tard, au moment où commença le débat sur ce sujet à l'Assemblée Générale des Nations Unies, l'Espagne constata l'apparition de catégories de filets dérivants de grande longueur (certains atteignant près de $55 \mathrm{~km}$ ) et ayant de grosses mailles, utilisés principalement pour capturer de grosses espèces pélagiques migratrices. La grande capacité de capture que ces filets possèdent, ajoutée au peu de séléctivité qui offrent les mailles utilisées, ${ }^{111}$ cause un impact très négatif sur l'ensemble de l'écosystème marin. De ce fait, le Ministère de l'Agriculture, de la Pêche et de l'Alimentation, adopta un décret le 22 octobre 1990, interdisant l'utilisation des engins de dérive, bien que l'on continue à tolérer leur emploi en tant qu'engin mineur dans le secteur méditérranéen. ${ }^{112}$

\footnotetext{
108 JOCE L 220 du 29 juillet 1982, p. 1 et ss. et JOCE L 207 du 29 juillet 1987, p. 1 et 11.

109 Voir supra note 74.

110 Arrêté du 24 novembre 1981, réglementant l'exercice de l'activité de pêche avec des engins fixes ou dérivants dans la Méditerranée, B.O.E. du 3 décembre 1981.

111 Cette pêche non sélective permet aussi bien la capture des espèces qui ne font pas l'objet de l'activité de pêche réalisée, que celle des espèces protégées, en danger d'extinction.

112 B.O.E. du 24 octobre 1990.
} 
Parallèlement, aussi bien au sein des Nations Unies que, plus tard, dans le cadre de l'Union européenne, l'Espagne a été l'un des Etats les plus actifs, et le plus ferme partisan de l'interdiction de l'emploi des filets dérivants. Au sein de l'Union européenne, l'utilisation de ces filets a fait l'objet de débats dans tous les Conseils des Ministres de la Pêche qui se sont réunis au cours de ces cinq dernières années, a l'occasion desquels, le Gouvernement espagnol a défendu son opposition à l'utilisation de ces filets. ${ }^{113}$

Suite à cela, l'Espagne présenta au Conseil des Ministres de la Pêche de l'Union européenne du 28 septembre 1994 une proposition visant à interdire complètement ce type d'engin à partir du 31 décembre 1994, son utilisation ne pouvant être autorisé qu'à l'intérieur de la zone côtière des 12 m.m. et, même ainsi, avec une longueur de filet individuelle ou cumulée non supérieure à 2,5 km. ${ }^{114}$ Malgré l'appui répété du Parlement Européen, ${ }^{115}$ cette proposition espagnole ne put pas compter sur des appuis suffisants au Conseil des Ministres de la Pêche. ${ }^{116}$ La proposition de la Commission d'interdire l'utilisation des filets dérivants et de maille à partir de 1998 fut ainsi maintenue. ${ }^{117}$

\subsection{Extraterritorialité de la loi interne espagnole}

Conformément à la jurisprudence internationale fermement ancrée depuis longue date, 118 la territorialité du droit pénal n'est pas un principe absolu du droit international. Les droits nationaux peuvent légitimement étendre l'action pénale aux délits commis en dehors du territoire étatique. Ce con-

113 Dans la comparution du Ministre espagnol de l'Agriculture, de la Pêche et de l'Alimentation au Congrès des Députés, le 14 septembre 1994, M. Atienza Serna résuma l'intense activité du gouvernement espagnol dans les forums Europeéns de la façon suivante: “Il faut rappeler que jusqu'en 1992 il n'y avait aucune limite pour les filets dérivants. Ces limites furent introduites par l'initiative du gouvernement espagnol dans le milieu de la Communauté européenne... Nous allons continuer sur cette voie, etant donné qu'il ne peut en être autrement, vu que c'est le secteur de pêche espagnol le plus intéréssé par la suppression des filets dérivants", ibidem, 14 septembre 1994, p. 4565 et ss.

114 Ibidem, p. 4550-4551.

115 A la même date, le Parlement Européen vota à la majorité (203 votes pour, 85 contre, 7 abstentions) une proposition de l'eurodéputée espagnole, Mme Carmen Fraga, qui coincide avec le contenu de la proposition présentée par l'Espagne au Conseil des Ministres de la Pêche. Ce fut la troisième fois où le Parlement Européen se manifesta en faveur de l'interdiction immédiate de ces filets. Auparavant, il l'avait fait dans le rapport à l'occasion de la publication du Règlement du Conseil n. 345/92 et aussi le 14 décembre 1993.

116 Seul la Grèce, et à un moindre niveau le Portugal, appuyèrent la proposition espagnole, et de ce fait il ne fut pas possible d'atteindre la majorité nécéssaire pour voter son approbation.

117 Dans sa comparution devant le Congrès des Deputés le 5 octobre 1994, le Ministre de l'Agriculture, de la Pêche et de l'Alimentation, M. Atienza Serna, affirma que, bien que le gouvernement espagnol juge de façon positive la proposition de la Commission, ce qui est un pas en avant vis-àvis de la situation actuelle, cela ne signifie pas que le gouvernement espagnol renonce à soutenir que les filets dérivants doivent être supprimés immédiatement.

118 Voir l'arrêt de la Cour Permanente de Justice Intemationale du 17 septembre 1927 dans le cas du Lotus, CPJI Publications, série A, n. 10, p. 20. 
cept a été appliqué dans la loi organique 6/1985 du 1er juillet, portant sur le Pouvoir Judiciaire, ${ }^{119}$ dont l'art. 23 par no. 1 a le contenu suivant:

"Dans l'ordre pénal, la connaissance des affaires concernant les délits et contraventions commis en territoire espagnol ou commis à bord de navires ou aéronefs espagnols, sans préjudice de ce qui est prévu dans les traités internationaux dont l'Espagne est partie, revient à la juridiction espagnole".

Mais outre l'extension de l'étendue de la juridiction pénale aux délits et contraventions commis à bord de navires et aéronefs espagnols, lorsque ceux-ci exercent les libertés de navigation et de survol de la haute mer, la juridiction pénale espagnole peut aussi s'étendre à la haute mer, pour la répression des délits contre le droit des gens, comme le stipule l'art. 23.4 de cette même loi organique:

"La juridiction espagnole sera également compétente pour connaître les faits, commis par des espagnols ou des étrangers en dehors du territoire national, susceptibles d'être qualifiés d'après la loi pénale espagnole comme l'un des délits suivants:

a) génocide

b) terrorisme

c) piraterie et appropriation illicite d'aéronefs

d) falsification de monnaies étrangères

e) actes relatifs à la prostitution

f) trafic illégal de substances psychotropiques, toxiques et stupéfiants

g) tout autre délit qui, d'après les traités ou accords internationaux doit être poursuivi en Espagne”.

Après cette ample énumération de délits contre le droit des gens, il faut rappeler que la piraterie et l'appropriation illicite d'aéronefs sont mentionnées dans les art. 100 à 107 de la Convention de Montego Bay, qui reconnaissent à tous les Etats le droit de poursuivre en haute mer les navires ou aéronefs pirates, d'arrêter les personnes et de confisquer les biens qui se trouvent à bord, de même que le droit de juger par leurs tribunaux internes les présumés pirates. En outre, l'Espagne a aussi adopté de règles sur le délit de piraterie. ${ }^{120}$ Elle est partie contractante à plusieurs accords internationaux qui cherchent à renforcer la sécurité de la navigation aérienne et maritime, et qui s'appliquent aussi en haute mer. Parmi les premiers, il convient de citer l'Accord sur les infractions et autres actes commis à bord d'aéronefs (Tokyo, 14 septembre 1963), ${ }^{121}$ l'Accord sur la répression de la capture illi-

119 B.O.E. du 2 juillet 1985; correction des erreurs dans le B.O.E. du 4 novembre 1985.

120 Voir les art. 138 et ss. du Code Pénal.

121 B.O.E. du 25 décembre 1969. 
cite d'aéronefs (La Haye, 16 décembre 1970) ${ }^{122}$ et l'Accord sur la répression d'actes illicites contre la sécurité de la navigation (Montréal, 23 septembre 1971). ${ }^{123}$ Parmi les seconds, il convient de citer la Convention pour la répression d'actes illicites contre la sécurité de la navigation maritime (Rome, 10 mars 1988) ${ }^{124}$ et son Protocole pour la répression d'actes illicites contre la sécurité des plates-formes fixes situées sur le plateau continental (Rome, 10 mars 1988). ${ }^{125}$

D'autre part, si l'art. 108 de la Convention de Montego Bay se limite à établir un devoir général pour tous les Etats de coopérer à la répression du trafic illicite de stupéfiants et de substances psychotropes réalisé par des navires en haute mer, la Convention des Nations Unies contre le trafic illicite de stupéfiants et de substances psychotropes (Vienne, 19 décembre 1988), dont l'Espagne est partie contractante, ${ }^{126}$ reconnaît de plus grands pouvoirs aux Etats. Ainsi, son art. 17 (consacré au trafic illicite en mer) stipule que lorsqu'un Etat soupçonne qu'un bateau qui arbore le pavillon d'un autre Etat se livre au trafic illicite en profitant de la liberté de navigation en haute mer, il pourra demander à l'Etat du pavillon l'autorisation d'aborder ce navire, de l'inspecter, et, s'il découvre des preuves d'implication dans le trafic illicite, d'adopter les mesures qui sont appropriées par rapport au navire, aux personnes et à la cargaison qui se trouvent à bord.

Un développement intéressant de cette Convention est représenté par le Traité entre le Royaume d'Espagne et la République Italienne pour la répression du trafic illicite de drogue en mer (Madrid, 23 mars 1990). ${ }^{127}$

Par cet accord bilatéral, chaque Etat partie se voit obligé de considérer comme délit dans son ordre interne une série de comportements en rapport avec le trafic maritime de stupéfiants ou de substances psychotropes, ${ }^{128}$ et à reconnaitre à l'autre un vaste droit d'intervention ${ }^{129}$ lorsqu'on soupçonne qu'à bord d'un bateau qui arbore le pavillon de l'autre partie se réalisent certains de ces comportements illégaux et, lorsque le bateau se trouve dans les eaux au-delà de la limite de la mer territoriale de son Etat, la possibilité d'abandon de juridiction de la part de l'Etat du pavillon en faveur de l'Etat qui a fait la capture est envisagée.

122 B.O.E. du 15 janvier 1973.

123 B.O.E. du 10 janvier 1974.

124 B.O.E. du 24 avril 1992.

125 Ibidem.

126 B.O.E. du 10 novembre 1990.

127 B.O.E. du 6 mai 1994.

128 Il s'agit de faits réalisés à bord de navires: à savoir la possession pour la distribution, le transport, le transbordement, le dépôt, la vente, la fabrication ou la transformation de stupéfiants ou de substances psychotropes (art. 2).

${ }^{129}$ D'après l'art. 5.2, les navires de guerre ou les aéronefs militaires ainsi que les autres navires et aéronefs de l'Etat de chaque partie pourront poursuivre, arrêter et aborder le bateau supçonné, vérifier les papiers, interroger les personnes qui se trouvent à bord, et si les soupçons sont fondés, inspecter le navire et, dans ce cas, procéder à la saisie de la drogue, à la mise aux arrêts des personnes impliquées et, s'il y a lieu, emmener le navire au port le plus proche, en informant immédiatement l'Etat du pavillon. 


\subsection{Navigation en haute mer}

Dans le droit espagnol, il n'existe pas de réglementation de la navigation internationale pour la haute mer. Il ne faut pas moins rappeler que l'Espagne participe à la quasi totalité des traités internationaux règlementant la sécurité de la navigation en mer, applicables aussi en haute mer. ${ }^{130}$

Quelques précisions dignes d'être mentionnées ont été introduites seulement à un niveau bilatéral, comme l'extension du Règlement international de 1972 afin d'empêcher les abordages en mer aux navires de guerre lorsque ceux-ci réalisent des manoeuvres ou des exercices militaires en haute mer, ${ }^{131}$ ou la reconnaissance du droit d'intervention en haute mer dans le but de combattre et d'arrêter le trafic international illicite de stupéfiants et de substances psychotropes. ${ }^{132}$

\section{ZONE INTERNATIONALE DES FONDS MARINS}

Durant les travaux du Comité des Fonds Marins et de la III CNUDM, l'attitude de la délégation espagnole fut fortement conditionnée par deux éléments de base: premièrement, par le fait que l'Espagne est un consommateur important et un producteur déficitaire des principales ressources minérales exploitables de la Zone internationale des fonds marins (nickel, cobalt, manganèse et cuivre), ce qui l'oblige nécessairement à les importer; deuxièmement, le fait tout aussi indiscutable que les entreprises espagnoles, publiques ou privées, n'avaient pas developpé la technologie nécessaire pour procéder elles-mêmes à l'exploitation des ressources minérales de la Zone.

La combinaison de ces deux facteurs rendit difficile l'identification et la défense des intérêts espagnols dans ce domaine au cours des très complexes négociations internationales. Si, en tant que pays consommateur et importateur de ces ressources minérales, l'Espagne était intéressée à la plus grande

\footnotetext{
130 L'Espagne fait partie des traités suivants: Convention pour l'unification de certaines règles en matière d'assistance et de sauvetage maritimes (Bruxelles, 23 septembre 1910) (G.M. du 13 décembre 1923); Convention sur les lignes de charge (Londres, 5 avril 1966), B.O.E. du 10 août 1968, 26 octobre 1968 et 1 septembre 1982; Convention sur le jaugeage des navires (Londres, 23 juin 1969), B.O.E. du 15 septembre 1982; Convention sur le règlement international pour prévenir les abordages en mer amendé (Londres, 20 octobre 1971), B.O.E. du 9 juillet 1977, 23 juin 1983 et 18 octobre 1984; Convention sur la securité des containers - amendé le 2 avril 1981 (Genève, 2 décembre 1972), B.O.E. du 13 septembre 1977 et 25 août 1982); Convention pour la sauvegarde de la vie humaine en mer amendé (Londres, 1 novembre 1974), B.O.E. du 16, 17 et 18 juin 1980 et 13 septembre 1980; Convention sur les normes de formation des gens de mer, de délivrance des brevets et de veille- amendé (Londres, 7 juillet 1978), B.O.E. du 7 novembre 1984 et 23 août 1994; Convention sur la recherche et le sauvetage maritime (Hambourg, 27 avril 1979), B.O.E. du 30 avril 1979 et du 21 septembre 1993; Convention pour la répression d'actes illicites contre la sécurité de la navigation maritime et Protocole pour la répression d'actes illicites contre la sécurité des plates-formes fixes situées sur le plateau continental (Rome, 10 mars 1988), B.O.E. du 24 avril 1992.

131 Voir supra, note 87.

132 Voir supra, note 127.
} 
exploitation des ressources de la Zone, son manque de développement technologique dans ce domaine faisait en sorte que, d'une certaine façon, il lui était indifférent que fût convenu un système d'exploration et d'exploitation des ressources de la zone.

Ceci explique en partie les hésitations de la délégation espagnole tout au long des négociations internationales. Considérant son faible développement technologique dans ce domaine, la délégation espagnole, dans un premier temps, adhéra aux idées défendues par le groupe des 77,133 avec l'espoir d'obtenir l'appui majoritaire de celui-ci sur d'autres questions qui la touchaient de façon plus directe. Plus tard, la divergence d'intérêts entre les Etats fortement industrialisés et les Etats du groupe des 77 s'accentuant, l'Espagne, mettant en avant sa condition de consommateur et d'importateur des ressources minerales de la Zone, proposa des formules de compromis visant à obtenir un authentique système parallèle qui fût opérationnel dans la pratique.

Lorsque les Etats fortement industrialisés et ceux du groupe des 77 parvinrent à un début d'accord sur la composition du Conseil et sur les obligations financières (financement du budget ordinaire de l'Autorité et de la première exploitation minière de l'entreprise), divers Etats moyennement industrialisés, au nombre desquels se trouvait l'Espagne, insistèrent, bien que sans succès, ${ }^{134}$ pour que leur forte contribution aux charges financières de l'Autorité et de l'Entreprise soit compensée par de plus grandes possibilités de représentation au Conseil.

La situation résultant de la III CNUDM ne satisfit pas complètement les intérêts de l'Espagne. D'un côté, le régime de la Partie XI de la Convention de Montego Bay, en plus de limiter sérieusement ses possibilités de représentation au Conseil, l'obligeait à assumer d'énormes charges financières sans garantir un résultat efficace, au vu de la non participation (surtout financière et technologique) des Etats fortements industrialisés. D'un autre côté, l'adoption par l'Espagne d'une législation unilatérale sur l'industrie minière des haut fonds marins, qu'avaient faite les Etats fortement industrialisés, n'avait aucun sens, étant donné que dans ce domaine, et en comparaison avec ces Etats, elle accusait un retard technologique évident.

Le rapprochement des positions entre les Etats fortement industrialisés et ceux du groupe des 77, lancé par le Secrétaire Général des Nations Unies et concrétisé dans l'Accord du 28 juillet 1994, relatif à l'application de la Partie XI de la Convention de Montego Bay, peut déterminer un changement positif de l'attitude espagnole vers le régime de cet espace maritime. L'introduction de principes d'économie de marché, la réduction des frais, la disparution de l'obligation de financer la première opération minière de

133 A la Commission des Fonds Marins, voir son intervention dans le Doc. A/AC.138/SC.I/SR.31, p. 211 ; voir son intervention dans III CNUDM, Documentos oficiales, I, p. 192 et ss..

134 Voir dans ce sens, l'amendement contenu dans le Doc. A/CONF.62/SR.175, parrainé par l'Espagne, et qui fut finalement retiré face à l'évidence de sa non approbation. 
l'entreprise, la mise en place progressive d'organes de l'Autorité, le renforcement du consensus dans l'adoption de décisions, etc., favorisent les intérêts espagnols dans ce domaine. S'il est vrai que les possibilités de représentation de l'Espagne au Conseil n'ont pas été touchées en profondeur, il est vrai aussi que la participation en principe des Etats fortement industrialisés à l'Accord de 1994 et les perspectives que le système soit opérationnel dans la pratique, n'est pas un élément étranger aux intérêts de l'Espagne. Ainsi, bien que les entreprises espagnoles, nonobstant un développement technologique constant, ne soient toujours pas en mesure de réaliser elles-mêmes une opération minière dans la zone, il n'y a pas de raison pour écarter une participation actionnaire ou une autre forme de participation dans un consortium minier international.

\section{DELIMITATION DES ZONES MARITIMES AVEC D'AUTRES ETATS}

\subsection{Attitude générale}

Le critère général suivi par l'Espagne pour délimiter ses espaces maritimes avec les Etats voisins, fut résumé comme suit par le Sous-Secrétaire des Affaires Etrangères, dans son intervention du 3 décembre 1991 devant la Commission des Affaires Etrangères:

"La conclusion, Messieurs, est, d'abord, que la politique en matière de délimitation des espaces maritimes avec les pays voisins est définie par le critèr général qui veut qu'on donne la priorité, là où il est possible, aux accords internationaux et, à défaut de ces accords, qu'on applique la ligne médiane ou la ligne équidistante. Des accords ont été conclus chaque fois que les raisons d'opportunité politique et les intérêts nationaux l'ont voulu. On déduit de tout cela...que d'une certaine façon, l'absence d'accords peut-être assimilée à l'existence d'un vide juridique". 135

L'attitude consistant à affirmer que les délimitations maritimes doivent être concrétisées dans un traité conclu avec l'Etat intéressé, et, qu'à défaut de ce traité, les espaces maritimes espagnols ne s'étendront pas au-delà de la ligne médiane ou ligne équidistante, apparaît dans la législation espagnole

\footnotetext{
135 B.O.C.G., IV Leg., Senado, Comisiones, n. 119, p. 2. Voir dans le même registre, la réponse du gouvernement espagnol dans B.O.C.G., III Leg., Congreso de Diputados, serie D, n. 315, p. 14830 et ss..
} 
sur la mer territoriale 136 et sur la ZEE. ${ }^{137}$ Cette ligne est aussi prescrite de façon conventionnelle pour la mer territoriale, la zone contiguë et le plateau continental espagnol. ${ }^{138}$ Elle correspond également à l'attitude défendue par la délégation espagnole au cours de la III CNUDM pour la délimitation du plateau continental et de la ZEE. 139

Il faut aussi signaler que dans les questions de délimitation des espaces maritimes espagnols, où la conclusion du traité en question n'a pas été possible, l'Espagne a toujours préféré appliquer le système de la ligne médiane ou ligne équidistante.

En bref, la politique espagnole en matière de délimitation de ses espaces maritimes reste ancrée au système genevois de 1958, ne tenant ainsi pas compte de l'importante évolution qui s'est produite dans ce domaine dans le droit international contemporain. Ainsi, l'Espagne continue à défendre la nécessité de conclure un accord pour fixer les frontières maritimes, en soutenant qu'à défaut d' accord, c'est le principe de l'équidistance qui prime. Elle continue, par ailleurs, à considérer que la présence des îles ne constitue pas chaque fois une circonstance spéciale qui pousserait à abandonner ce principe. Cependant, l'application de la règle de l'équidistance, à défaut d'accord de délimitation, n'apporte pas toujours une solution acceptable. En effet, plusieurs des Etats voisins dont les côtes sont contiguës, ou font face à celles de l'Espagne, n'acceptent pas la règle de l'équidistance comme norme subsidiaire pour la délimitation des espaces marins.

\subsection{Accords de délimitation}

Très peu de délimitations d'espaces maritimes espagnols contigus ou opposés à ceux des autres Etats ont fait l'objet d'un accord international. Il faut rappeller, en premier lieu, la délimitation des eaux intérieures convenue entre l'Espagne et la France pour le Bidasoa et la Baie de Higuer. Depuis

\footnotetext{
136 L'art. 4 de la loi 10/1977 du 4 janvier, sur la mer territoriale cit., a le contenu suivant: "Excepté un accord mutuel qui s'y opposerait, la mer territoriale ne s'étendra pas, en rapport avec les pays voisins et avec ceux dont les côtes se trouvent face aux côtes espagnoles, au-delà d'une ligne médiane déterminée de telle façon que tous ses points soient équidistants des points les plus proches des lignes de base à partir desquelles on mesure la largeur de la mer territoriale de chacun desdits Etats, et qui sont tracées en conformité avec le droit international". Voir également le par. 6 de l'exposé des motifs de cette loi.

137 D'après l'art. 2 de la loi 15/1978 du 20 février sur la ZEE, cit., : "1. Excepté ce qui est dit dans les traités internationaux avec les Etats dont les côtes se trouvent en face des côtes espagnoles ou sont adjacentes à celles-ci, la limite extérieure de la zone économique sera la ligne de milieu ou ligne équidistante. 2. En relation avec cet article, on entend par ligne du milieu ou équidistante, celle dont les points sont équidistants des points les plus proches situés dans les lignes de base tracées en conformité avec le droit international, à partir desquelles la largeur de la mer territoriale de chaque Etat est mesurée...".

138 Voir les art. 12.1 et 24.3 de la Convention de 1958 sur la mer territoriale (B.O.E. du 24 décembre 1971) et l'art. 6 de la Convention de 1958 sur le plateau continental (B.O.E. du 25 décembre 1971).

139 Durant la III CNUDM, l'Espagne soutint le groupe d'Etats partisans de la règle de l'équidistance, corrigée par les circonstances spéciales, fàce aux Etats partisans du recours aux principes d'équité.
} 
très longtemps, ${ }^{140}$ les deux pays s'étaient mis d'accord pour tracer une ligne de fermeture de la baie. Cette ligne était divisée en trois segments égaux à partir desquels furent tracées des lignes droites vers les côtes les plus proches, entraînant ainsi la création de trois zones, l'une française, l'autre espagnole, et une au milieu en condominium des deux Etats.

Des accords de délimitation de la mer territoriale ont été conclus, l'un avec la France dans le Golfe de Biscaye (Paris, 29 janvier 1974), ${ }^{141}$ qui a recours au méridien (pour les premiers $6 \mathrm{~m} . \mathrm{m}$.) associé à l'équidistance (pour les 6 m.m. suivants) et l'autre avec le Portugal dans l'embouchure du fleuve Minho (27 mars 1893), suivant le parallèle du milieu du canal d'embouchure du fleuve.

Des accords de délimitation du plateau continental ont été conclus avec la France dans le Golfe de Biscaye (Paris, 29 janvier 1974142 qui combine la ligne d'équidistance avec l'existence de circonstances spéciales, vu qu'il s'agit de la plus grande longueur de la côte françaises par rapport à la côte espagnole, et qui établit par ailleurs une zone d'exploitation commune); et avec l'Italie (Madrid, 19 février 1974), ${ }^{143}$ qui suit une ligne d'équidistance pure entre les îles de Minorque et de Sardaigne.

Le 12 février 1976, l'Espagne et le Portugal signèrent à Guarda deux accords concernant la délimitation d'espaces maritimes: le premier porte sur la délimitation de la mer territoriale et de la zone contiguë (à cette date, de 6 à $12 \mathrm{~m} . \mathrm{m}$. respectivement pour chaque Etat); le deuxième porte sur la délimitation du plateau continental. ${ }^{144}$ Dans les deux accords, les lignes de délimitation de la mer territoriale, de la zone contiguë et du plateau continental ne furent pas les lignes d'équidistance: dans le cas du fleuve Minho, la frontière maritime suit le parallèle qui passe par le milieu de l'embouchure; dans le cas du fleuve Guadiana, la frontière maritime fut tracée suivant le méridien qui traverse le milieu de l'embouchure. Au début de 1978, les deux Etats entamèrent des négociations pour délimiter leur ZEE à 1'intérieur de leur deux frontières péninsulaires, de même que pour la délimitation du plateau continental et de la ZEE entre Madère et les Iles Canaries.

Bien qu'un début d'accord pour les frontières péninsulaires fut rapidement atteint, la survenance de circonstances économiques et juridiques fut

\footnotetext{
140 Voir supra, note 9.

141 B.O.E. du 4 juillet 1975; voir également l'Echange de Lettres hispano-français du 29 janvier 1974, sur l'interprétation de l'art. 2 b) du dit Accord (B.O.E. du 9 juillet 1975).

142 B.O.E. du 9 juillet 1975.

143 B.O.E. du 5 décembre 1978.

${ }^{144}$ Le texte des deux Accords peut-être consulté dans: B.O.C.G., n. 1512 du 15 juin 1976, p. 36553-36556.
} 
la cause du refus portugais de ratifier les deux accords signés en 1976 à Guarda. ${ }^{145}$ Ces accords n'entreront probablement jamais en vigueur.

\section{PRESERVATION ET PROTECTION DE L'ENVIRONNEMENT MARIN}

\subsection{Attitude générale}

Etant donné le nombre croissant d'accidents maritimes survenus en mer territoriale espagnole, en particulier à proximité des côtes de Galice et de la corniche Cantabrique, ainsi que l'importante industrie du tourisme et de la pêche qui se concentre sur le littoral espagnol, la grande importance que l'Etat espagnol attache à la préservation et à la protection du milieu marin n'a rien d'étonnant. Cette importance était déjà évoquée dans la première intervention (29 juillet 1971) portant sur ce sujet de la délégation espagnole à la Commission des Fonds Marins. On y soutenait que "le devoir de protéger le milieu marin et d'éliminer la menace de sa pollution" est "une règle fondamentale que la délégation espagnole n'hésite pas à qualifier de véritable règle de jus cogens, dans le sens que cette expression a dans la Convention de Vienne de 1969 sur le droit des traités". 146

Le délégué espagnol ajoutait que, d'après lui, la méthode la plus appropriée à suivre était celle d'un traitement global, plutôt que sectoriel, des problèmes de la pollution du milieu marin. ${ }^{147}$ Cette affirmation retient l'attention, car l'Espagne n'a jamais eu de loi pour la protection de l'environnement en général, ni de loi générale limitée à la protection du milieu marin. En outre, dans la législation de base sur les différents espaces mariti-

\footnotetext{
145 Les circonstances économiques survenues correspondaient à la découverte d'un gisement de gaz sur le plateau continental se trouvant à l'embouchure du fleuve Guadiana, justement dans une zone qui, d'après les accords de Guarda ferait partie du plateau continental espagnol, mais qui, en se basant sur les critères de l'équidistance, appartiendrait au plateau continental portugais. Ceci explique probablement que le Portugal ait voulu fixer unilatéralement les limites externes de sa ZEE, en se basant uniquement sur les critères de l'équidistance (décret-loi portugais 119/1978 du ler juin). Le 16 août 1978, l'Espagne contesta cette délimitation unilatérale en allèguant qu'elle ne coïncidait pas avec les délimitations convenues dans les Accords de Guarda de 1976 et produisait des chevauchements entre les ZEE et les plateaux continentaux des deux Etats. L'Espagne contesta également le dit décret-loi portugais en soutenant que le Portugal voulait créer une ZEE autour des Iles Sauvages, situées entre Madère et les Iles Canaries, bien qu'il s'agisse de rochers inhabités.

146 Intervention du délégué espagnol à la Commission des Fonds Marins (III Sous-Commission). Reproduite dans DE YTURRIAGA BARBERAN, Textos... cit., supra note 1, pp. 499-500.

147 Ibidem, p. 498.
} 
mes espagnols, les références à la protection de l'environnement sont, selon les cas, inexistantes, ${ }^{148}$ indirectes, ${ }^{149}$ ou absentes. ${ }^{150}$

Ainsi, la législation espagnole sur la préservation et la protection du milieu marin se trouve à la fois dans un grand nombre de traités internationaux portant sur ce thème, dont l'Espagne est partie, et dans un vaste ensemble de dispositions du droit interne espagnol ayant un statut légal très varié. 151

La loi 27/1992 du 24 novembre sur les ports de l'Etat et la Marine Marchande $152 \mathrm{n}$ 'en fait pas moins un effort important pour donner un cadre juridique à caractère global à toute cette réglementation. Ainsi son art. 60, dont il faut regretter la rédaction défectueuse, à le contenu suivant:

“1. Les déversements ou émissions polluants, qu'ils soient solides, liquides ou gazeux, dans le secteur portuaire public, provenant de navires ou de moyens flottants de n'importe quelle sorte sont interdits...

2. Les déversements polluants provenant de navires ou moyens flottants de n'importe quelle sorte, dans les eaux non portuaires dans lesquelles l'Espagne exerce sa souveraineté, ses droits souverains ou sa juridiction, seront réglementés par des normes spécifiques applicables et par les accords souscrits par l'Espagne concernant ces matières.

3. Tous les déversements de la terre à la mer requièrent l'autorisation de l'Administration compétente qui sera accordée en fonction de la législation applicable".

\subsection{Pollution provenant des navires}

En ce qui concerne la pollution incidente ou opérationnelle provenant de navires, l'Espagne accepta le 22 janvier 1964 la Convention pour la prévention de la pollution des eaux de la mer par les hydrocarbures (Londres, 12 mai 1954). ${ }^{153}$ Ses dispositions avaient été auparavant appliquées dans le

148 Il n'existe aucune mention à la protection de l'environnemnt dans la loi 10/1977 du 4 janvier, sur la mer territoriale espagnole. Supra, note 7.

149 D'après l'art. 1.2, b) à $d$ ) de la loi 15/1978 du 20 février, sur la ZEE (supra, note 11), l'Etat espagnol a "la compétence pour réglementer la conservation et l'exploitation de ces ressources, pour prendre soin de la préservation du milieu marin; la juridiction exclusive pour faire respecter les dispositions pertinentes; pour toutes les autres compétences que le gouvernement établit en conformité avec le droit international".

150 Comme c'est le cas des eaux intérieures ou du plateau continental espagnol.

151 Parfois cette diversité normative est favorisée par la Constitution espagnole elle-même. Rappelons par exemple que son art. 149.1.23ème affirme la compétence exclusive de l'Etat en matière de législation de base sur la protection de l'environnement, “au détriment des facultés des Communautés Autonomes d'établir des normes supplémentaires de contrôle", alors que son art. 148.1.9ème, soutient que les Communautés Autonomes peuvent assumer des compétences en rapport avec la gestion en matière de protection de l'environnement.

152 Voir supra note 13

153 Cette Convention fut revisée le 13 avril 1962, introduisant des zones où la décharge d'hydrocarbures était interdite. Le texte de la Convention fut publié dans le B.O.E. du 29 juillet 1964. 
droit espagnol. ${ }^{154}$ Les amendements à cette Convention, adoptés le 21 octobre 1969, furent acceptés par l'Espagne par l'acte du 13 janvier 1976.155

Cette Convention fut remplacée par un texte beaucoup plus ambitieux: la Convention pour la prévention de la pollution par les navires (Londres, 2 novembre 1973), modifiée par le Protocole du 17 février 1978, connu comme la Convention MARPOL. L'acte espagnol de ratification de la Convention MARPOL ${ }^{156}$ spécifia que l'Espagne n'acceptait aucune obligation prévue aux annexes facultatives de cette Convention. Sur les cinq annexes, l'Espagne n'acceptait que l'Annexe I, portant les règles pour éviter la pollution par hydrocarbures et l'Annexe II, qui porte les règles pour éviter la pollution par des substances nocives liquides transportées en vrac. Plus tard, par l'acte du 27 décembre 1990, l'Espagne accepta l'Annexe III (règles relatives à la prévention de la pollution par substances nuisibles transportées par mer, en colis, containers, citernes mobiles et camions ou wagons citernes); ${ }^{157}$ l'Annexe IV (règles relatives à la prévention de la pollution par les eaux sales des navires), et l'Annexe $\mathrm{V}$ (règles pour éviter la pollution par les déchets des navires). ${ }^{158}$ L'Espagne a accepté aussi la plupart des amendements approuvés pour la Convention MARPOL. 159

Plus récemment, et suite à la Convention MARPOL, la loi 27/1992 du 24 novembre sur les Ports de l'Etat et la Marine Marchande, ${ }^{160}$ après avoir interdit les émissions polluantes provenant "de navires ou de moyens flottants de n'importe quelle sorte", précise que les restes ou résidus solides et liquides provenant de sentines, de ballasts, de lavages de citernes ou de caves, d'huiles usées, d'eaux sales et d'autres liquides polluants, devront être déchargés à terre et déposés dans des récipients, des installations ou des citernes que l'Autorité Portuaire aura choisis (art. 60.1).

Pour cela, les raffineries de pétrole, les usines chimiques et pétro-chimiques, les installations de stockage et de distribution de produits chimiques

\footnotetext{
154 Par deux décrets ministériels du ler juin 1963, le premier portant sur les normes de construction de navires afin d'éviter la pollution des eaux de mer par les hydrocarbures, et le second sur les normes pour éviter la pollution des eaux de mer par hydrocarbures (publiés au B.O.E. du 6 juin 1963) et, aussi, au décret du 24 septembre 1963 interdisant aux navires la décharge en mer des résidus de citemes de combustibles (B.O.E. du 3 octobre 1963).

155 B.O.E. du 27 janvier 1978. Ces amendements furent appliqués par le décret ministériel du 30 décembre 1977 sur les décharges d'hydrocarbures par les navires (B.O.E. du 14 février 1977).

156 Acte du 22 juin 1984, B.O.E. du 17 et 18 octobre 1984.

157 B.O.E. du 6 mars 1991 et du 25 mai 1992.

158 Les Annexes IV et V sont publiées dans le B.O.E. du 6 mars 1991.

159 Les amendements approuvés en 1984 sont publiés dans le B.O.E. du 3 décembre 1985. Les amendements du 5 décembre 1985 dans le B.O.E. du 27 janvier 1987; ceux du ler décembre 1987 dans le B.O.E. du 10 avril 1989; ceux du 17 mars 1989 dans le B.O.E. du 27 septembre 1990; les amendements de l'Annexe V du 17 octobre 1989 dans le B.O.E. du 21 janvier 1991, etc. En Espagne, la Convention MARPOL a été développée par le décret du 18 décembre 1984 sur les normes de séparateurs et oléomètres (B.O.E. du 5 février 1985 et correction des erreurs dans le B.O.E. du 23 mars 1985) et par le décret du 26 mars 1985 sur l'interdiction du transport d'hydrocarbures ou autres substances inflammables pour le milieu marin (B.O.E. du 30 mars 1985).
}

160 Voir supra note 13. 
ou pétro-chimiques, les installations pour le ravitaillement de combustibles liquides qui possèdent des terminaux de charge ou de décharge d'hydrocarbures dans les zones portuaires et les chantiers navals et installations de réparations navales, devront disposer aux environs des terminaux ou des quais, d'installations pour la réception et le traitement des déchets pétroliers et chimiques et des eaux de sentine et pour le nettoyage des huiles des graisses et autres produits polluants, de même que des moyens nécéssaires pour éviter et combattre les écoulements (art. 61.1). En relation avec cette obligation, on édicta le décret royal 438/1994 du 11 mars, qui réglemente l'installation de réception de déchets huileux provenant des navires, en application de la Convention MARPOL. ${ }^{161}$

En ce qui concerne la pollution accidentelle par les navires, l'Espagne ratifia, le 19 février 1973, la Convention sur l'intervention en haute mer en cas d'accident entraînant ou pouvant entraîner une pollution par hydrocarbures (Bruxelles, 29 novembre 1969);162 elle devint également partie au Protocole sur l'intervention en haute mer en cas de pollution par des substances autres que les hydrocarbures (Londres, 2 novembre 1973) ${ }^{163}$ et au Protocole relatif à la coopération en matière de lutte contre la pollution de la Mer Méditerranée par les hydrocarbures et autres substances nuisibles en cas de situation critique (Barcelone, 16 février 1976). ${ }^{164}$

En complément à cette réglementation, et dans un but également préventif de la pollution accidentelle, l'Espagne a pris plusieurs mesures internes de protection. Au nombre de celles-ci, l'on retiendra la nécessité d'une autorisation préalable pour permettre la navigation des navires à propulsion nucléaire, ou des navires qui transportent des substances nucléaires dans la mer territoriale espagnole. ${ }^{165}$ Elle a aussi établi les conditions minimales afin que certains navires-citernes entrent ou sortent des ports espagnols. ${ }^{166}$ Elle a même interdit la détention et le mouillage des navires-citernes dans la mer territoriale et dans la ZEE. ${ }^{167}$

Il faut également signaler que l'Espagne a signé, (sa ratification étant en cours), aussi bien la Convention sur la coopération, la préparation et la lutte contre la pollution par hydrocarbures (Londres, 30 novembre 1990), que la Convention sur la coopération pour la protection des côtes et des eaux de l'Atlantique du Nord-Est contre la pollution (Lisbonne, 17 octobre 1990). D'autre part, la loi 27/1992 du 24 novembre, sur les ports de l'Etat et la Marine Marchande, 168 a prévu l'obligation du Gouvernement d'approuver un Plan national de services spéciaux de sauvetage de vies humaines en

\footnotetext{
161 B.O.E. du 8 avril 1994.

162 B.O.E. du 26 février 1976.

163 B.O.E. du 2 mai 1994.

164 B.O.E. du 21 février 1978.

165 Voir supra note 36.

166 Décret du 8 novembre 1990. Voir supra note 18

167 Décret du 17 avril 1991. Voir supra note 28.

168 Voir supra note 13.
} 
mer et de lutte contre la pollution du milieu marin (art. 87.2) dont l'objectif de base est: de coordonner le rôle des différents moyens capables de réaliser des opérations de recherche, de sauvetage de vies humaines et de lutte contre la pollution marine, appartenant aux différentes Administrations, ainsi qu'à des institutions publiques ou privées; d'établir un système de contrôle du trafic maritime qui englobe la totalité de nos côtes, et ce, par la mise en place de Centres Coordinateurs Régionaux et Locaux; d'augmenter les moyens de sauvetage et de lutte contre la pollution marine déjà existants, et de former un personnel spécialisé qui sera responsable de la direction et de la coordination des opérations de recherche, de sauvetage et de lutte contre la pollution marine. Ce plan national fut accepté le 11 mars 1993.

Il faut aussi signaler que l'Espagne est partie à la Convention sur la responsabilité civile pour les dommages dus à la pollution par hydrocarbures (Bruxelles, 29 novembre 1969)169 et à son Protocole (Londres, 19 novembre 1976); 170 à la Convention portant création d'un fond international d'indemnisation pour les dommages dus à la pollution par hydrocarbures (Bruxelles, 18 décembre 1971) $)^{171}$ et à son Protocole (Londres, 19 novembre 1976), ${ }^{172}$ et à la Convention relative à la responsabilité civile dans le domaine du transport maritime de matières nucléaires (Bruxelles, 17 décembre 1971).173 L'Espagne a commencé la procédure d'autorisation parlementaire pour adhérer au Protocole d'amendement à la Convention sur la responsabilité civile pour les dommages dus à la pollution par hydrocarbures (Londres, 27 novembre 1992) et au Protocole d'amendement à la Convention portant création d'un fond international d'indemnisation pour les dommages dus à la pollution par les hydrocarbures (Londres, 27 novembre 1992).

De son côté, la loi 27/1992 du 24 novembre sur les ports de l'Etat et la Marine Marchande a érigé le non respect des dispositions en vigueur sur les éléments, les installations et les documents à bord pour la prévention et le contrôle des opérations d'évacuation des déchets et autres substances, en une infraction grave; ${ }^{174}$ alors que l'évacuation des déchets et autres matières provenant directement ou indirectement des opérations normales des navires, plates-formes fixes ou autres constructions qui se trouvent sur des eaux situées dans des zones où l'Espagne exerce sa souveraineté, droits

169 B.O.E. du 8 mars 1976.

170 B.O.E. du 4 février 1992.

171 B.O.E. du 11 mars 1982.

172 Ratifié par l'Espagne par l'acte du 5 avril 1982. Vu que ce Protocole n'est toujours pas entré en vigueur, on ne l'a pas publié dans le B.O.E.

173 B.O.E. du 20 août 1975.

174 Art. 115.4 c), supra note 13. 
souverains ou juridiction, est considérée comme infraction grave ou très grave selon que l'évacuation soit involontaire ou délibérée. ${ }^{175}$

\subsection{Immersion des déchets en mer}

L'Espagne est partie à plusieurs traités internationaux réglementant cette source de pollution: la Convention sur la prévention de la pollution des mers résultant de l'immersion de déchets (Londres, Mexico D.F, Moscou et Washington, 29 décembre 1972), qui est applicable à toutes les mers, excepté les eaux intérieures; 176 la Convention pour la prévention de la pollution marine par les opérations d'immersion effectuées par les navires et aéronefs (Oslo, 15 février 1972), qui est applicable dans la zone de l'Atlantique Nord-Est; 177 et le Protocole relatif à la prévention de la pollution de la Mer Méditerranée par les opérations d'immersion effectuées par les navires et aéronefs (Barcelone, 16 février 1976). ${ }^{178}$

Les Conventions de Londres et Oslo furent introduites dans le droit espagnol par un décret de 1976, applicable à tous les navires et aéronefs espagnols, de même qu'aux navires et aéronefs étrangers qui se trouvent dans toute zone maritime soumise à la souveraineté ou à la juridiction espagnole. ${ }^{179} \mathrm{Ce}$ décret interdit le déversement en mer de certaines substances, exige l'obtention d'une autorisation préalable spécifique des autorités compétentes pour le déversement en mer d'autres substances, et requiert une autorisation des autorités compétentes pour le déversement en mer des substances restantes. ${ }^{180} \mathrm{La}$ loi $21 / 1977 \mathrm{du}$ ler avril, concernant l'application de sanctions dans le cas de pollution marine provoquée par des déversements provenant de navires ou aéronefs, ${ }^{181}$ établit les sanctions applicables

175 Ibidem, art. $115.4 a$ ) et 116.4 c). Au cas où il n'y aurait pas lieu d'appliquer une sanction pénale, la sanction administrative des infractions graves pour contamination du milieu marin consiste en une amende pouvant aller jusqu'à 100 millions de pesetas; si l'infraction est très grave, la somme de l'amende peut aller jusqu'à 500 millions de pesetas; a cettes sommes on peut ajouter, comme sanctions complémentaires, la détention des navires ou empêcher son entrée, soit les opérations de déchargement de celui-ci. Ibidem, art. 119 et 120. Il existe d'autres mesures qui n'ont pas un caractère de sanction, telle que l'obligation de remettre les choses là où elles étaient, ou celle de verser des indemnités pour les dégâts irréparables, etc. Ibidem, art. 121.

176 L'Espagne le ratifia par l'acte du 31 juillet 1974, publié dans le B.O.E. du 10 novembre 1975. L'Amendement de la liste des substances, approuvé par la résolution LDC 12 (V) du 24 septembre 1980, est publié dans le B.O.E. du 6 mai 1981. L'amendement adopté par la résolution LDC 5 (III) du 12 octobre 1978 sur l'incinération en mer fut publié dans le B.O.E. du 7 novembre 1980.

177 Ratifié par l'Espagne par l'acte du 14 juin 1973, et publié dans le B.O.E. du 25 avril 1974. Le 2 mars 1983 il fut adopté un Protocole d'amendement par lequel sont introduites "des normes obligatoires d'incinération en mer". L'Espagne adhèra à ce Protocole d'amendement par l'acte du 15 juin 1989, publié dans le B.O.E. du 27 septembre 1989.

178 B.O.E. du 21 février 1978.

179 Art. 6 du Décret du 26 mai 1976 sur la prévention de la pollution marine causée par des déversements provenant de navires et aéronefs, B.O.E. du 4 juin 1976. L'art. 7 sauvegarde "l'immunité dont jouissent certains navires et aéronefs, en accord avec le droit international".

180 Ibidem, art. 2,3 et 4.

181 B.O.E. du 4 avril 1977. 
dans le cas d'infractions aux dispositions sur le déversement et désigne les Autorités compétentes pour les appliquer. Récemment, la loi 27/1992 du 24 novembre sur les ports de l'Etat et la Marine Marchande a abrogé la loi 21/1977 “où l'on fait référence à l'application de sanctions en cas de pollution marine causée par des déversements provenant de navires"182 afin d'unifier les sanctions applicables aux différentes sources de pollution. En vertu de celle-ci, on considère comme infraction très grave, aussi bien l'évacuation délibérée par des navires ou des plates-formes fixes ou autres constructions qui se trouvent dans des zones maritimes espagnoles, de déchets, résidus ou autres matières, entreposées à bord ou déposées dans ce but, que le naufrage de navires ou la destruction de plates-formes fixes ou autres constructions, excepté lorsqu'on dispose de l'autorisation exigée ou que celle-ci n'est pas obligatoire d'après la législation spécifique en vigueur. ${ }^{183}$

\subsection{Pollution tellurique}

L'Espagne est partie contractante à la Convention pour la prévention de la pollution marine d'origine tellurique (Paris, 21 février 1974) ${ }^{184}$ et au Protocole sur la protection de la Mer Méditerranée contre la pollution d'origine terrestre (Athènes, 17 mai 1980). 185

Le régime juridique espagnol sur le déversement de la terre à la mer de substances dangereuses est défini notamment ${ }^{186}$ par l'Instruction pour le déversement en mer, depuis la terre, des eaux résiduelles à travers des émetteurs sous marins ${ }^{187}$ (où sont établies deux listes de substances qui, à cause de leur bio-accumulabilité, toxicité et persistance, doivent être fortement limitées dans les effluents), et par la loi 22/1988 du 28 juillet relatives aux côtes. ${ }^{188}$

182 Disposition dérogatoire unique, $2 q$ ).

183 Art. $116.4 a$ ) et $b$ ). Voir supra note 175.

184 Ratifié par l'Espagne par l'acte du 27 novembre 1980 et publié dans le B.O.E. du 21 janvier 1981.

185 Ratifié par l'Espagne par l'acte du 21 mai 1984 et publié au B.O.E. du 26 juin 1984. L'Annexe IV de ce Protocole est au B.O.E. du 10 décembre 1993. Bien qu'en principe chaque convention ait pour but d'empêcher la pollution marine d'origine terrestre, elles couvrent cependant un large éventail de sources de pollution marine, incluant aussi bien la pollution d'origine terrestre par l'atmosphère (dans le cas de la Convention de Paris, cette source de pollution fut introduite par le Protocole d'amendement du 26 mars 1986, publié au B.O.E. du 14 mai 1990), que les déchargements polluants provenant de structures artificielles fixes installées en mer, qui sont sous la juridiction d'un Etat partie.

186 Certaines d'entre elles sont antérieures aux deux conventions à peine citées, comme le décret du 27 mai 1967, relatif à l'interdiction de déverser en mer des produits pétrolifères ou des déchets contaminés, qui établit, dans son art. 1, l'interdiction aux usines et aux industries de tout type, de déverser en mer des produits pétrolifères ou des déchets qui contiennent des substances pétrolifëres, aussi bien persistantes que non-persistantes (B.O.E. du 1er juin 1967).

187 Approuvée par décret ministériel du 29 avril 1977, B.O.E. du 25 juin 1977.

188 B.O.E. du 29 juillet 1988 . Voir également le décret royal 1471/1989 du ler décembre, par lequel le règlement général pour le développement et l'exécution de la loi 22/1988 du 28 juillet, de côtes est approuvé (B.O.E. du 12 décembre 1989). 
Conformément à cette dernière réglementation, le déversement de déchets solides et de décombrements en mer est interdit. Pour le déversement d'autres substances, on exige l'autorisation préalable de l'administration compétente. ${ }^{189}$ De manière générale, on ne peut déverser des substances ni introduire des formes d'énergie qui peuvent représenter un danger ou un préjudice supérieur à ce qui est toléré par la santé publique et l'environnement, et cela doit être fait conformément à la législation en vigueur. Même pour les déversements qui n'ont pas de conséquences aussi graves, on exige leur limitation "dans la mesure où le permet l'état de la technique, les matières premières et, en particulier, en tenant compte de la capacité d'absorption de la charge polluante, sans qu'une altération significative ne se produise" du milieu récepteur de la pollution (art. 56 et 57).

Le programme général, applicable expressément à la pollution tellurique, complète ces dispositions. D'après ce programme, grâce aux opérations de gestion des déchets toxiques et dangereux, on évitera de déplacer la pollution ou la détérioration de l'environnement vers un autre milieu récepteur. ${ }^{190}$

D'autre part, afin d'incorporer dans la législation espagnole les règles du droit dérivé européen, ${ }^{191}$ fut édicté le décret royal 258/1989 du 10 mars, portant réglementation générale sur les déversements de substances dangereuses de la terre à la mer. Ce décret royal fixe les normes applicables à tout déversement, effectué de la terre vers les eaux intérieures et en mer territoriale espagnole, susceptible de contenir une ou plusieurs substances dangereuses, en se basant sur les limites maximales d'émission pour éliminer la pollution par déversement de substances de la liste I (c'est à dire les substances les plus toxiques, persistantes et bio-accumulables) et sur des objectifs de qualité, pour réduire la pollution par déversement de substances de la liste II (autres substances moins préjudiciables). ${ }^{192}$

\section{RECHERCHE SCIENTIFIQUE MARINE}

Le décret royal 799/1981 du 27 février ${ }^{193}$ porte les règles applicables aussi bien aux escales dans les ports espagnols de navires étrangers se consacrant à la recherche scientifique marine et qui ne mènent, et ne prétendent

\footnotetext{
$189 \mathrm{Il}$ incombe à l'Administration de l'Etat d'accorder l'autorisation des déversements, sauf industriels et exceptés les polluants de la terre à la mer; les Communautés Autonomes exercent les compétences qui, en matière de déversements, leur sont attribuées en vertu de leur statut respectif (art. 110 et 114 de la loi).

190 Art. 6.3 de la loi 20/1986 du 14 mai, sur le régime juridique de base des déchets toxiques et dangereux, B.O.E. du 20 mai 1986.

191 Fondamentalement, la directive du Conseil 76/464/CEE du 4 mai 1976 relative à la pollution causée par des substances dangereuses déversées dans le milieu aquatique de la Communauté et la normative générale établie dans la directive du Conseil 86/280/CEE du 12 juin 1986.

192 Art. 1., B.O.E. du 16 mars 1989.

193 B.O.E. du 8 mai 1981.
} 
pas mener, de telles activités dans des zones soumises à la juridiction espagnole, qu'à la réalisation d'activités de recherche scientifique marine dans les zones soumises à la juridiction espagnole.

Dans le premier cas, on exige l'autorisation préalable du Ministère espagnol des Affaires Etrangères qui en informera, à son tour, le Ministère de la Défense. Le Ministère des Affaires Etrangères communiquera expressément à l'Etat demandeur si l'escale demandée est autorisée et les conditions qui, le cas échéant, sont à remplir.

Dans le deuxième cas, aussi bien les Etats tiers que les organisations internationales qui prétendent mener des activités de recherche scientifique marine dans la mer territoriale, la ZEE ou le plateau continental espagnol, devront obtenir l'autorisation du Ministère espagnol des Affaires Etrangères. Ce Ministère pourra exiger de l'Etat demandeur qu'il complète l'information présentée avec les renseignements que l'on estime nécessaires pour la meilleure détermination du programme de recherche. Ce sera également ce Ministère qui fera savoir à l'Etat demandeur si on autorise ou non la campagne proposée, les conditions requises dans ce cas, et si on souhaite ou non la participation de scientifiques espagnols à celle-ci. L'autorisation devra être délivrée expressément pour la mer territoriale. En revanche, elle peut être obtenue suite à un silence administratif d'une période de quatre mois pour les recherches dans d'autres zones maritimes espagnoles.

L'exercice d'activités de recherche scientifique marine qui sont menées dans la ZEE ou sur le plateau continental peut entraîner l'arrêt de celles-ci, si elles ne se font pas en accord avec l'information transmise dans le programme de recherche par l'Etat demandeur, ou si la réglementation espagnole en vigueur n'est pas respectée, et si les conditions prévues par l'autorisation sont violées. La suspension des activités peut se transformer en une obligation de cessation si ces manquements impliquent un changement important du projet ou des activités scientifiques projetées.

Quelle que soit la zone maritime espagnole dans laquelle ces activités se réalisent, l'Etat demandeur devra toujours remettre au Ministère espagnol des Affaires Etrangères les résultats de la campagne ainsi que l'ensemble des renseignements obtenus, de même que la copie des publications et travaux scientifiques ou tous autres travaux qui en découlent.

\section{BIBLIOGRAPHIE}

ABELLAN HONRUBIA, Espanya i el nou Dret del Mar, 1988;

ALBIOL BIOSCA, El régimen jurídico de los fondos marinos internacionales, 1984;

AMAYA TURRIENTES et JUSTE RUIZ, La politica agrícola y de pesca en la Comunidad Europea, 1986; 
ASIN CABRERA, Islas y archipiélagos en las Comunidades Europeas. Estudio de los regímenes jurídicos especiales con particular consideración de Canarias, 1988;

AZCARRAGA BUSTAMANTE, Derecho del Mar, 2 vol., 1983;

BAEZA BETANCORT, Las islas Canarias ante el nuevo Derecho internacional del mar, 1987;

BOU FRANCH, La navegación por el mar territorial, incluidos los estrechos internacionales y las aguas archipelágicaś, en tiempos de paz, 1994;

CABRERA CALVO SOTELO et MACAU I NADAL, La Europa azul, 1986;

CASADO RAIGON, La pesca en alta mar, 1994.

CERVERA PERY, La problemática de la pesca en el nuevo Derecho del mar. Ideas para su conocimiento, 1984;

CERVERA PERY, El Derecho del Mar. Evolución, contenido, perspectivas (de las bulas papales al Convenio de Jamaica), 1992;

CORRAL SUAREZ, El vertido de residuos radioactivos en la "fosa atlántica" y el Derecho internacional, 1988;

DE YTURRIAGA BARBERAN, Regional Conventions on the Protection of the Marine Environment, RC, 1978, IV, p. 321-449;

DE YTURRIAGA BARBERAN, Straits Used for International Navigation: A Spanish Perspective, 1991;

DE YTURRIAGA BARBERAN, Ambitos de soberanía en la Convención de las Naciones Unidas sobre el Derecho del Mar. Una perspectiva española, 1993;

JIMENEZ PIERNAS, La revisión del estatuto territorial del Estado por el nuevo Derecho del Mar: el caso de los Estados archipelágicos, 1990;

JUSTE RUIZ et CASTILLO DAUDI, La pesca alicantina en el actual proceso de revisión del Derecho del Mar, 1983;

LACLETA, PASTOR RIDRUEJO et DE YTURRIAGA BARBERAN, $L a$ III Conferencia de las Naciones Unidas sobre el Derecho del Mar, 1978; 
MARTINEZ PUÑAL, Los derechos de los Estados sin litoral y en situación geográfica desventajosa en la zona económica exclusiva (Participación en la explotación de los recursos vivos), 1988;

ORIHUELA CALATAYUD, España y la delimitación de sus espacios marinos, 1989;

PARDOS, Actuaciones diplomáticas de España en los vertimientos de residuos nucleares radioactivos, 1983;

PASTOR RIDRUEJO, La explotación de los fondos marinos más allá de la jurisdicción nacional, 1975;

PEREZ PEREZ, Lucha contra la contaminación por vertidos de hidrocarburos, 1993;

POCH (sous la direction de), La actual revisión del Derecho del Mar: Una perspectiva española, 1975, 4 vol.;

PUEYO LOSA, El archipiélago oceánico. Regulación jurídico-maritima internacional, 1981;

RIQUELME CORTADO, España ante la Convención sobre el Derecho del Mar. Las declaraciones formuladas, 1990;

SANCHEZ RODRIGUEZ, La zona exclusiva de pesca en el muevo Derecho del mar, 1977;

SANCHEZ RODRIGUEZ, España y el régimen internacional de la pesca maritima, 1986;

SUAREZ DE VIVERO, El nuevo orden oceánico. Consecuencias territoriales, 1985;

SUAREZ DE VIVERO, Las aguas interiores en la ordenación del litoral, 1992;

URUEÑA ALVAREZ, La protección del medio marino en España, 1986. 